MATHEMATICS OF COMPUTATION

Volume 75 , Number 253 , Pages 43-71

S 0025-5718(05)01778-3

Article electronically published on September 29, 2005

\title{
SHARP $L^{1}$ A POSTERIORI ERROR ANALYSIS FOR NONLINEAR CONVECTION-DIFFUSION PROBLEMS
}

\author{
ZHIMING CHEN AND GUANGHUA JI
}

\begin{abstract}
We derive sharp $L^{\infty}\left(L^{1}\right)$ a posteriori error estimates for initial boundary value problems of nonlinear convection-diffusion equations of the form

$$
\frac{\partial u}{\partial t}+\operatorname{div} f(u)-\Delta A(u)=g
$$

under the nondegeneracy assumption $A^{\prime}(s)>0$ for any $s \in \mathbf{R}$. The problem displays both parabolic and hyperbolic behavior in a way that depends on the solution itself. It is discretized implicitly in time via the method of characteristic and in space via continuous piecewise linear finite elements. The analysis is based on the Kružkov "doubling of variables" device and the recently introduced "boundary layer sequence" technique to derive the entropy error inequality on bounded domains. The derived a posteriori error estimators have the correct convergence order in the region where the solution is smooth and recover the standard a posteriori error estimators known for parabolic equations with strong diffusions.
\end{abstract}

\section{INTRODUCTION}

A posteriori error estimates are computable quantities in terms of the discrete solution and data that measure the actual discrete errors without the knowledge of exact solutions. The adaptive finite element method based on a posteriori error estimates initiated in 3 provides a systematic way to refine or coarsen the mesh according to the local a posteriori error estimators on the elements. There are considerable efforts in the literature devoted to the development of a posteriori error analysis and efficient adaptive algorithms for various linear and nonlinear parabolic partial differential equations (see, e.g., 17, 21, 7, 5, 8, and the references therein).

Let $\Omega$ be a bounded domain in $\mathbf{R}^{d}(d=1,2,3)$ with Lipschitz boundary and $T>0$. In this paper, we consider the sharp a posteriori error analysis for the following nonlinear convection-diffusion equation:

$$
\frac{\partial u}{\partial t}+\operatorname{div} f(u)-\Delta A(u)=g \quad \text { in } Q
$$

Received by the editor September 3, 2004 and, in revised form, September 29, 2004.

2000 Mathematics Subject Classification. Primary 65N15, 65N30, 65N50.

This author was supported in part by China NSF under grant 10025102 and by China MOST under grant G1999032802 and 2005CB321700. Part of the work was done when the first author was participating in the 2003 Programme Computational Challenges in Partial Differential Equations at the Isaac Newton Institute for Mathematical Sciences, Cambridge, United Kingdom.

(C)2005 American Mathematical Society 
with the initial and boundary conditions

$$
\left.u\right|_{t=0}=u_{0},\left.\quad u\right|_{\partial \Omega \times(0, T)}=0 .
$$

Here $u=u(x, t) \in \mathbf{R}$, with $(x, t) \in Q=\Omega \times(0, T)$. We assume that the function $f$ : $\mathbf{R} \rightarrow \mathbf{R}^{d}$ is locally Lipschitz continuous, the function $A: \mathbf{R} \rightarrow \mathbf{R}$ is nondecreasing and locally Lipschitz continuous, $g \in L^{\infty}(Q)$ and $u_{0} \in L^{\infty}(\Omega)$.

Problems of the type (1.1) model a wide variety of physical phenomena including porous media flow, flow of glaciers, and sedimentation processes [24]. Our motivation comes from the simulation of flow transport through unsaturated porous media which is governed by the so-called Richards equation [2, 22, where $x=\left(x_{1}, \ldots, x_{d}\right)$,

$$
\frac{\partial S}{\partial t}+\frac{\partial K(S)}{\partial x_{d}}-\operatorname{div}(K(S) \nabla p)=0
$$

where $S$ is the volumetric water content, $p$ is the pressure head, and $K(S)$ is the relative permeability. One of the widely used nonlinear constitutive relations for $S=S(p)$ and $K=K(S)$ in the engineering literature, the so-called van GenuchtenMualem formula, reads as follows:

$$
S(p)=\left(1+|\alpha p|^{n}\right)^{-m}, \quad K(S)=S^{1 / 2}\left(1-\left(1-S^{1 / m}\right)^{m}\right)^{2},
$$

where $m=1-1 / n, \alpha>0, n>1$ are shape constants which vary for different types of porous media. For (1.3), the existence of weak solutions is considered in [2] and the uniqueness of weak solutions is proved in 29] based on Kružkov's "doubling of variables" technique. Entropy solutions for (1.1) are studied in [4] and [27. The mathematical techniques developed in 27] play an important role in the analysis in this paper.

Our discretization of (1.1) is based on combining continuous piecewise linear finite elements in space with the characteristic finite difference in time. The method of characteristic originally proposed in [16, 31] is widely used to solve convectiondiffusion problems in the finite element community (cf., e.g., 22, 1, 21, 8, 23]). Given $U_{h}^{n-1}$ as the finite element approximation of the solution at time $t^{n-1}$, let $\tau_{n}$ and $V_{0}^{n} \subset H_{0}^{1}(\Omega)$ be the time step and the conforming linear finite element space at the $n$th time step. Then our discrete scheme reads as follows: find $U_{h}^{n} \in V_{0}^{n}$ such that

$$
\left\langle\frac{U_{h}^{n}-\bar{U}_{h}^{n-1}}{\tau_{n}}, v\right\rangle+\left\langle\nabla A\left(U_{h}^{n}\right), \nabla v\right\rangle=\left\langle\bar{g}^{n}, v\right\rangle \quad \forall v \in V_{0}^{n},
$$

where $\bar{g}^{n}=\tau_{n}^{-1} \int_{t^{n-1}}^{t^{n}} g(x, t) d t, \bar{U}_{h}^{n-1}(x)=U_{h}^{n-1}\left(\tilde{X}\left(t^{n-1}\right)\right)$, and the approximate characteristic $\tilde{X}(t)$ is defined by

$$
d \tilde{X} / d t=f^{\prime}\left(U_{h}^{n-1}(\tilde{X}(t))\right), \quad \tilde{X}\left(t^{n}\right)=x .
$$

In the linear case when $f(u)=\mathbf{v} u, A(u)=\epsilon u$ for some small constant $\epsilon>0$, and the $L^{2}\left(L^{2}\right)$ a posteriori error estimate is proved in 21] based on the duality argument. A priori error analysis for the method of characteristic can be found, for example, in [16, 31, 13, 1.

The well-known Kružkov "doubling of variables" technique originally appeared in 26] and plays a decisive role in the error estimation (both a posteriori and a priori) for numerical schemes solving the Cauchy problems of nonlinear conservation laws (see, e.g., 10, 11, 12, 25] and the reference therein). It was also used recently in 28] for the implicit vortex centered finite volume discretization of the Cauchy problems 
of (1.1) for general nonnegative $A^{\prime}(s) \geq 0$ for all $s \in \mathbf{R}$. The common feature of these studies is that the derived error indicators are of the order $\sqrt{h}$ in the region where the solution is smooth, where $h$ is the local mesh size. We remark that in the region where the diffusion is dominant, the error indicators developed for the parabolic equations (cf., e.g., [30, 7]) are of order $h$. Thus the degeneration of the order of the error indicators used in 28] may cause over-refinements for the solution of (1.1) in the region where the diffusion is dominant. We also refer to 20] for a different approach of a posteriori error estimation for nonlinear conservation laws and to the recent work 19, 18 for developing a convergent finite volume scheme for solving nonlinear degenerate parabolic equations.

The basic assumption in this paper is that the diffusion is positive:

$$
A^{\prime}(s)>0, \quad \forall s \in \mathbf{R} .
$$

This assumption includes the Richards equation (1.3) and the viscosity regularization of degenerate parabolic equations, for example, the regularized continuous casting problem which is considered in [8. The novelty of our analysis with respect to the analysis for nonlinear conservation laws in [11, 12, 25] or nonlinear degenerate parabolic equations in [28] lies in the following aspects. First, only Cauchy problems are considered in 11, 12, 25, 28. The difficulty of including the boundary condition is essential. In this paper, we use the recently introduced technique of "boundary layer sequence" in [27] to overcome the difficulty. We remark that the use of the technique of "boundary layer sequence" allows us to extend the analysis in the paper to treat other types of boundary conditions, in particular, the nonhomogeneous Dirichlet boundary conditions. We will report the progress in this respect in future studies. We emphasize that even for homogeneous boundary conditions, the technique of "boundary layer sequence" is important, as it allows us to truncate the standard Kružkov test function (see (4.6) below) to obtain the admissible test function in the entropy error identity (see Section 4 for details). Second, the nature of the estimators are different: our estimators emphasize the diffusion effect of the problem which requires the assumption $A^{\prime}(s)>0$ for any $s \in \mathbf{R}$; the estimates in [28] are valid for any nonlinear function $A$ such that $A^{\prime}(s) \geq 0$. The nice consequence of the analysis in this paper is that our a posteriori error estimates are able to recover the standard sharp a posteriori error estimators in the literature derived for a parabolic problem with diffusion coefficients bounded uniformly away from zero (see Remark 5.8). Further remarks about the differences of the a posteriori error estimates in this paper from those in 11, 12, 25, 28, can be found in Remark 5.10,

The rest of the paper is organized as follows. In Section 2 we set the notation and briefly recall the definition of entropy solutions for (1.1). In Section 3 we introduce the discrete problem. In Section 4 we derive the important entropy error inequality by using boundary layer sequence technique. In Section 5 we derive the a posteriori error estimates and present several remarks. In Section 6 we report two numerical examples.

\section{Setting}

Let $\Omega$ be a bounded domain in $\mathbf{R}^{d}$ with Lipschitz boundary. Defining $\mathcal{B}$ as the set of all possible Lipschitz coverings of $\partial \Omega$ in the sense that $\partial \Omega \subset \bigcup_{B \in \mathcal{B}} B$, and, in some local coordinates $x=\left(x^{\prime}, x_{d}\right)$, there exists a Lipschitz function $x_{d}=\rho\left(x^{\prime}\right)$ 
such that $B \cap \partial \Omega=\left\{x \in B: x_{d}=\rho\left(x^{\prime}\right)\right\}, B \cap \Omega=\left\{x \in B: x_{d}<\rho\left(x^{\prime}\right)\right\}$. Given $T>0$, let $Q=\Omega \times(0, T)$. We start by stating the hypotheses concerning the data.

(H1) $f: \mathbf{R} \rightarrow \mathbf{R}^{d}$ is locally Lipschitz continuous, $f(0)=0 ; f^{\prime}$ is globally Lipschitz continuous in $\mathbf{R}$.

(H2) $A: \mathbf{R} \rightarrow \mathbf{R}$ is locally Lipschitz continuous, $A(0)=0 ; A^{\prime}(s)>0$ for any $s \in \mathbf{R}$, and $A^{\prime} \circ A^{-1}$ is globally Hölder continuous in $\mathbf{R}$, i.e.,

$$
\left|\left(A^{\prime} \circ A^{-1}\right)(s)-\left(A^{\prime} \circ A^{-1}\right)(r)\right| \leq C|s-r|^{\gamma} \quad \forall s, r \in \mathbf{R},
$$

for some constant $0<\gamma \leq 1$ and some constant $C>0$.

(H3) $g \in L^{\infty}(Q), u_{0} \in L^{\infty}(\Omega)$.

We recall the following definition of entropy solutions to the problem (1.1)-(1.2) in [27.

Definition 2.1. A function $u \in L^{\infty}(Q)$ is an entropy solution of the problem (1.1)-(1.2) if:

(i) (regularity) we have

$$
A(u) \in L^{2}\left(0, T ; H_{0}^{1}(\Omega)\right),
$$

and, for every $B \in \mathcal{B}$, and any nonnegative $\psi \in C_{c}^{\infty}(B)$, we have (here $x=\left(x^{\prime}, x_{d}\right)$, $x_{d}=\rho\left(x^{\prime}\right)$ on $\left.B \cap \partial \Omega\right)$

$$
(-|u| \psi, \operatorname{sgn}(u)(\nabla A(u)-f(u)) \psi) \in \mathcal{D} \mathcal{M}(Q)_{2},
$$

where $\mathcal{D} \mathcal{M}(Q)_{2}$ is the set of measure-divergence vector fields in $Q$ defined by

$$
\begin{aligned}
& \mathcal{D M}(Q)_{2}=\left\{(w, v) \in L^{2}(Q) \times L^{2}(Q)^{d}: \exists C>0\right. \text { such that } \\
& \left.\qquad\left|\int_{Q}\left(w \varphi_{t}+v \nabla \varphi\right) d x d t\right| \leq C\|\varphi\|_{L^{\infty}(Q)} \quad \forall \varphi \in C_{c}^{\infty}(Q)\right\} ;
\end{aligned}
$$

(ii) (entropy condition in the interior of $Q$ ) $u$ is an entropy solution of the equation with test functions zero on the boundary, i.e.,

$$
\begin{gathered}
-\int_{Q}|u-k| \partial_{t} \phi-\int_{Q} \operatorname{sgn}(u-k)[f(u)-f(k)-\nabla A(u)] \cdot \nabla \phi \\
\leq \int_{Q} \operatorname{sgn}(u-k) g \phi
\end{gathered}
$$

for any $\phi \in H_{0}^{1}(Q), \phi \geq 0$, for any $k \in \mathbf{R}$;

(iii) (initial condition) the initial condition is assumed to be the limit in $L^{1}$ sense,

$$
\operatorname{esslim}_{t \rightarrow 0^{+}} \int_{\Omega}\left|u(x, t)-u_{0}(x)\right| d x=0 .
$$

Note that since $A^{\prime}(s)>0$ for any $s \in \mathbf{R}$ by (H2), the entropy boundary condition in [27] is automatically satisfied [27, Remark 1.2], and thus we have not included it in the above definition of entropy solutions for (1.1)-(1.2). The main implication of the regularity property (2.1) lies in that it provides a proper meaning of the normal trace of the vector $(-|u| \psi, \operatorname{sgn}(u)(\nabla A(u)-f(u)) \psi)$ on the boundary. Since our analysis does not involve properties of measure-divergence vector fields, we refer the interested readers to [27] and the reference therein for further discussion on measure-divergence vector fields. It is proved in [27] that (1.1)-(1.2) has a unique entropy solution $u$ in the sense of Definition 2.1. Another definition of entropy solutions for (1.1) -(1.2) can be found in [4]. 
By taking $k>\operatorname{essup}_{Q} u(x, t)$ and $k<\operatorname{essinf}_{Q} u(x, t)$ in (2.2), it is easy to see that an entropy solution is also a weak solution of the same problem in the following sense.

Definition 2.2. A function $u$ is a weak solution of the problem (1.1)-(1.2) if

$$
\partial_{t} u \in L^{2}\left(0, T ; H^{-1}(\Omega)\right), \quad f(u) \in L^{2}\left(0, T ; L^{2}(\Omega)\right), \quad A(u) \in L^{2}\left(0, T ; H_{0}^{1}(\Omega)\right),
$$

and

$$
\int_{0}^{T}\left\langle\partial_{t} u, \varphi\right\rangle d t+\int_{Q}(-f(u)+\nabla A(u)) \cdot \nabla \varphi d x d t=\int_{Q} g \varphi d x d t
$$

for any $\varphi \in L^{2}\left(0, T ; H_{0}^{1}(\Omega)\right)$ such that $\phi(\cdot, 0)=\phi(\cdot, T)=0$. The initial condition is assumed to be the limit in $L^{1}$ sense as in (2.3). Here $\langle\cdot, \cdot\rangle$ stands for either the inner product in $L^{2}(\Omega)$ or the duality pairing between $H^{-1}(\Omega)$ and $H_{0}^{1}(\Omega)$.

The existence of weak solutions follows directly from the existence of entropy solutions. Since $A$ is strictly increasing we know that the weak solution $u$ of (2.4) is also unique. By a straightforward application of the weak maximum principle we have $u \in L^{\infty}(Q)$ (cf., e.g., [27, Lemma 2.4]). Then standard theory for parabolic equations implies that $\partial_{t} u \in L^{2}(Q)$. Consequently, by (1.1), $\Delta A(u) \in L^{2}(Q)$. We summarize these results in the following theorem.

Theorem 2.3. Let the hypotheses (H1)-(H3) be satisfied. Then there exists a unique weak solution $u$ to (1.1)-(1.2). Moreover, the following regularity results are valid:

$$
u \in L^{\infty}(Q), \quad \partial_{t} u \in L^{2}(Q), \quad \Delta A(u) \in L^{2}(Q) .
$$

We remark that if the domain $\Omega$ has a smooth boundary or $\Omega$ is convex, then we can obtain the regularity $u \in L^{2}\left(0, T ; H^{2}(\Omega)\right)$ by the standard regularity theory for parabolic equations. We also remark that the symbol $u$ will denote the weak solution of (1.1)-(1.2) throughout the paper.

\section{Discretization}

We now introduce the fully discrete problem, which combines continuous piecewise linear finite elements in space with the characteristic finite difference method in time. In fact, we use the method of characteristic to discretize the convection [16, 31, 22, 1, 21, 23. We denote by $\tau_{n}$ the $n$-th time step and set

$$
t^{n}:=\sum_{i=1}^{n} \tau_{i}, \quad \varphi^{n}(\cdot):=\varphi\left(\cdot, t^{n}\right)
$$

for any function $\varphi$ continuous in $\left(t^{n-1}, t^{n}\right]$. Let $N$ be the total number of time steps, that is, $t^{N} \geq T$. If

$$
d X(t) / d t=f^{\prime}(u(X(t), t)), \quad X\left(t^{n}\right)=x,
$$

defines the backward characteristic in $\left(t^{n-1}, t^{n}\right)$, then $U(t)=u(X(t), t)$ satisfies

$$
d U(t) / d t=\partial_{t} u+f^{\prime}(u) \cdot \nabla u=\partial_{t} u+\operatorname{div} f(u) .
$$

The characteristic finite difference method is based on writing

$$
\bar{x}^{n-1}=X\left(t^{n-1}\right), \quad \bar{u}^{n-1}(x)=u\left(\bar{x}^{n-1}, t^{n-1}\right)
$$


for $n \geq 1$ and discretizing (3.2) by means of backward differences as follows:

$$
\frac{d U^{n}}{d t} \approx \frac{U^{n}-U^{n-1}}{\tau_{n}} \Longrightarrow \partial_{t} u^{n}+\operatorname{div} f\left(u^{n}\right) \approx \frac{u^{n}-\bar{u}^{n-1}}{\tau_{n}}
$$

Therefore, the discretization in time of (1.1) reads

$$
\frac{u^{n}-\bar{u}^{n-1}}{\tau_{n}}-\Delta A\left(u^{n}\right)=\bar{g}^{n} \quad \text { in } \Omega,
$$

where $\bar{g}^{n}(x)=\tau_{n}^{-1} \int_{t^{n-1}}^{t^{n}} g(x, t) d t$. Since $\bar{u}^{n-1}$ is well defined only for $\bar{x}^{n-1} \in \bar{\Omega}$, one has to properly extend $u^{n-1}$ outside the domain according to the boundary condition imposed. For the homogeneous boundary condition, for example, if $\bar{x}^{n-1}=x_{0}+d^{n-1} \nu_{0}$ is outside the domain $\Omega$, where $x_{0} \in \partial \Omega, \nu_{0}$ the unit outer normal of $\partial \Omega$ at $x_{0}$, and $d^{n-1}$ the distance between $\bar{x}^{n-1}$ and $x_{0}$, then one may take $\bar{u}^{n-1}(x)=u^{n-1}\left(\bar{x}^{n-1}\right):=-u\left(x_{0}-d^{n-1} \nu_{0}, t^{n-1}\right)$.

Let $\mathcal{M}^{n}$ be a shape-regular triangulation of $\Omega$ into simplexes. The mesh $\mathcal{M}^{n}$ is obtained by refinement/coarsening of $\mathcal{M}^{n-1}$, and thus $\mathcal{M}^{n}$ and $\mathcal{M}^{n-1}$ are compatible. Two meshes are compatible if one is the local refinement by bisection of the other. For any $K \in \mathcal{M}^{n}, h_{K}$ stands for its diameter. We also denote $\mathcal{B}^{n}$ as the collection of sides $e$ of $\mathcal{M}^{n}$ in $\Omega ; h_{e}$ stands for the size of $e \in \mathcal{B}^{n}$.

Let $V^{n}$ indicate the usual space of continuous piecewise linear finite elements over $\mathcal{M}^{n}$ and let $V_{0}^{n}=V^{n} \cap H_{0}^{1}(\Omega)$. Let $U_{h}^{0} \in V^{n}$ be some discretization of the initial function $u_{0}$ so that $\left\|u_{0}-U_{h}^{0}\right\|_{L^{1}(\Omega)}$ can be arbitrarily small upon sufficient refinement of the initial mesh $\mathcal{M}_{0}$.

Discrete problem. Given $U_{h}^{n-1} \in V^{n-1}$, then $\mathcal{M}^{n-1}$ and $\tau_{n-1}$ are modified to get $\mathcal{M}^{n}$ and $\tau_{n}$. Thereafter $U_{h}^{n} \in V_{0}^{n}$ is computed according to

$$
\left\langle\frac{U_{h}^{n}-\bar{U}_{h}^{n-1}}{\tau_{n}}, v\right\rangle+\left\langle\nabla A\left(U_{h}^{n}\right), \nabla v\right\rangle=\left\langle\bar{g}^{n}, v\right\rangle \quad \forall v \in V_{0}^{n},
$$

where $\bar{U}_{h}^{n-1}=U_{h}^{n-1}\left(\bar{x}^{n-1}\right), \bar{x}^{n-1}=\tilde{X}\left(t^{n-1}\right)$, and the approximate characteristic $\tilde{X}(t)$ is defined by

$$
d \tilde{X} / d t=f^{\prime}\left(U_{h}^{n-1}(\tilde{X}(t))\right), \quad \tilde{X}\left(t^{n}\right)=x .
$$

The characteristic $\tilde{X}(t)$ satisfying (3.5) can be calculated by multistep Euler method or Runge-Kutta method as suggested in [1] or [21. If the time-step size $\tau_{n}$ is small enough (depending on the boundedness of $U_{h}^{n-1}$ ), then due to (H1) it can be easily proved that the approximate characteristics do not cross each other (cf., e.g., [23]). In this paper, we will not elaborate on this issue and simply assume this to be the case and still denote by $\tilde{X}(t)$ this approximate characteristic. Further details on the application of the method of characteristic to the nonlinear convection-diffusion problem can be found in [22, 23].

We also remark that since $A(\cdot)$ is strictly monotone, (3.4) can be solved by nonlinear SOR [8] if appropriate mass lumping is used for computing $\left\langle U_{h}^{n}, v\right\rangle$ for $v \in V^{n}$, and the nonlinear relation $A\left(U_{h}^{n}\right)$ is enforced node-wise, i.e., to replace $\left\langle\nabla A\left(U_{h}^{n}\right), \nabla v\right\rangle$ in (3.4) by $\left\langle\nabla I^{n} A\left(U_{h}^{n}\right), \nabla v\right\rangle$, where $I^{n}: C(\bar{\Omega}) \rightarrow V^{n}$ is the standard finite element Lagrange interpolant. The a posteriori error analysis below can be easily extended to cover these situations by including appropriate error indicators for quadrature error. To avoid inessential complications, we will not consider the extensions in this paper. 
We conclude this section with some notation. Let the jump of $\nabla A\left(U_{h}^{n}\right)$ across some $e \in \mathcal{B}^{n}$ be

$$
\llbracket \nabla A\left(U_{h}^{n}\right) \rrbracket_{e}:=\left(\left.\nabla A\left(U_{h}^{n}\right)\right|_{K_{1}}-\left.\nabla A\left(U_{h}^{n}\right)\right|_{K_{2}}\right) \cdot \nu_{e}
$$

with the convention that the unit normal vector $\nu_{e}$ to $e$ points from $K_{2}$ to $K_{1}$ and so that the jump $\llbracket \nabla A\left(U_{h}^{n}\right) \rrbracket_{e}$ is well defined. Let $U_{h}$ denote the piecewise linear extension of $\left\{U_{h}^{n}\right\}$, that is, $U_{h}(\cdot, 0)=U_{h}^{0}(\cdot)$, and for all $t^{n-1}<t \leq t^{n}$,

$$
U_{h}(\cdot)=\frac{t^{n}-t}{\tau_{n}} U_{h}^{n-1}(\cdot)+\frac{t-t^{n-1}}{\tau_{n}} U_{h}^{n}(\cdot)
$$

Finally, we introduce the mesh-dependent norms

$$
\begin{gathered}
\left\|h_{n} \varphi\right\|_{L^{2}(\Omega)}=\left(\sum_{K \in \mathcal{M}^{n}}\left\|h_{K} \varphi\right\|_{L^{2}(K)}^{2}\right)^{\frac{1}{2}}, \\
\left\|h_{n}^{1 / 2} \varphi\right\|_{L^{2}(\Omega)}=\left(\sum_{e \in \mathcal{B}^{n}} h_{e}\|\varphi\|_{L^{2}(e)}^{2}\right)^{\frac{1}{2}} .
\end{gathered}
$$

\section{ENTROPY ERROR INEQUALITY}

We start by introducing some notation. For any $\varepsilon>0$, let

$$
H_{\varepsilon}(z)=\operatorname{sgn}(z) \min (1,|z| / \varepsilon)
$$

be the regularization of the sign function $\operatorname{sgn}(z)$. For any $k \in \mathbf{R}$, define the entropy pair $\left(U_{\varepsilon}, F_{\varepsilon}\right)$ by

$$
U_{\varepsilon}(z, k)=\int_{k}^{z} H_{\varepsilon}(A(r)-A(k)) d r, \quad F_{\varepsilon}(z, k)=\int_{k}^{z} H_{\varepsilon}(A(r)-A(k)) f^{\prime}(r) d r .
$$

The following result is well known (cf., e.g., [4, 27]).

Lemma 4.1. For any $\phi \in L^{2}\left(0, T ; H_{0}^{1}(\Omega)\right)$ such that $\phi(\cdot, 0)=\phi(\cdot, T)=0$, and any $k \in \mathbf{R}$, we have

$$
\begin{gathered}
-\int_{Q} U_{\varepsilon}(u, k) \partial_{t} \phi-\int_{Q} F_{\varepsilon}(u, k) \cdot \nabla \phi+\int_{Q} H_{\varepsilon}(A(u)-A(k)) \nabla A(u) \cdot \nabla \phi \\
+\int_{Q} H_{\varepsilon}^{\prime}(A(u)-A(k))|\nabla A(u)|^{2} \phi=\int_{Q} g H_{\varepsilon}(A(u)-A(k)) \phi .
\end{gathered}
$$

By letting $\epsilon \rightarrow 0$ in (4.1) one obtains the entropy condition in the interior of $Q$ (2.2). In this paper, however, we will not use this limit interior entropy condition.

Let $\left(H^{1}(\Omega)\right)^{\prime}$ be the dual space of $H^{1}(\Omega)$. We define the discrete residual $\mathcal{R} \in$ $L^{2}\left(0, T ;\left(H^{1}(\Omega)\right)^{\prime}\right)$ through the following relation, for any $\varphi \in H^{1}(\Omega)$ :

$$
\left\langle\partial_{t} U_{h}, \varphi\right\rangle-\left\langle f\left(U_{h}\right), \nabla \varphi\right\rangle+\left\langle\nabla A\left(U_{h}\right), \nabla \varphi\right\rangle=\langle g, \varphi\rangle-\langle\mathcal{R}, \varphi\rangle .
$$

Then similar to Lemma 4.1, we have the following result. 
Lemma 4.2. For any $\phi \in L^{2}\left(0, T ; H_{0}^{1}(\Omega)\right)$ such that $\phi(\cdot, 0)=\phi(\cdot, T)=0$, and any $k^{\prime} \in \mathbf{R}$, we have

$$
\begin{gathered}
(4.3)-\int_{Q} U_{\varepsilon}\left(U_{h}, k^{\prime}\right) \partial_{t} \phi-\int_{Q} F_{\varepsilon}\left(U_{h}, k^{\prime}\right) \cdot \nabla \phi+\int_{Q} H_{\varepsilon}\left(A\left(U_{h}\right)-A\left(k^{\prime}\right)\right) \nabla A(u) \cdot \nabla \phi \\
\quad+\int_{Q} H_{\varepsilon}^{\prime}\left(A\left(U_{h}\right)-A\left(k^{\prime}\right)\right)\left|\nabla A\left(U_{h}\right)\right|^{2} \phi \\
=\int_{Q} g H_{\varepsilon}\left(A\left(U_{h}\right)-A\left(k^{\prime}\right)\right) \phi-\int_{0}^{T}\left\langle\mathcal{R}, H_{\varepsilon}\left(A\left(U_{h}\right)-A\left(k^{\prime}\right)\right) \phi\right\rangle .
\end{gathered}
$$

Proof. For the sake of completeness, we sketch the proof here. We take $\varphi=$ $H_{\varepsilon}\left(A\left(U_{h}\right)-A\left(k^{\prime}\right)\right) \phi$ in (4.2), integrate in time over $(0, T)$, and rewrite each term as follows. First, by integration by parts, we get

$$
\int_{0}^{T}\left\langle\partial_{t} U_{h}, H_{\varepsilon}\left(A\left(U_{h}\right)-A\left(k^{\prime}\right)\right) \phi\right\rangle=\int_{Q} \partial_{t} U_{\varepsilon}\left(U_{h}, k^{\prime}\right) \phi=-\int_{Q} U_{\varepsilon}\left(U_{h}, k^{\prime}\right) \partial_{t} \phi .
$$

Next, let $\psi_{\varepsilon}\left(z, k^{\prime}\right)=\partial_{z}\left[H_{\varepsilon}\left(A(z)-A\left(k^{\prime}\right)\right)\right]=H_{\varepsilon}^{\prime}\left(A(z)-A\left(k^{\prime}\right)\right) A^{\prime}(z)$. Then it is easy to see that

$$
\begin{aligned}
F_{\varepsilon}\left(z, k^{\prime}\right) & =\int_{k^{\prime}}^{z} H_{\varepsilon}\left(A(r)-A\left(k^{\prime}\right)\right) f^{\prime}(r) d r \\
& =f(z) H_{\varepsilon}\left(A(z)-A\left(k^{\prime}\right)\right)-\int_{k^{\prime}}^{z} f(r) \psi_{\varepsilon}\left(r, k^{\prime}\right) d r
\end{aligned}
$$

Thus, by doing integration by parts, we have

$$
\begin{aligned}
& -\int_{0}^{T}\left\langle f\left(U_{h}\right), \nabla \varphi\right\rangle \\
= & -\int_{Q}\left(\psi_{\varepsilon}\left(U_{h}, k^{\prime}\right) \nabla U_{h} \cdot f\left(U_{h}\right) \phi+H_{\varepsilon}\left(A\left(U_{h}\right)-A\left(k^{\prime}\right)\right) f\left(U_{h}\right) \cdot \nabla \phi\right) \\
= & -\int_{Q} F_{\varepsilon}\left(U_{h}, k^{\prime}\right) \cdot \nabla \phi-\int_{Q} \operatorname{div}\left(\phi \int_{k^{\prime}}^{U_{h}} f(r) \psi_{\varepsilon}\left(r, k^{\prime}\right) d r\right) \\
= & -\int_{Q} F_{\varepsilon}\left(U_{h}, k^{\prime}\right) \cdot \nabla \phi .
\end{aligned}
$$

The rest of the proof is straightforward, and we omit the details.

Now we are going to apply the Kružkov "doubling of variables" technique and will always write $u=u(y, s), U_{h}=U_{h}(x, t)$, unless otherwise stated. If necessary, in the following we will write $Q_{(x, t)}$ or $Q_{(y, s)}$ to stress the domain of integration with respect to $(x, t)$ or $(y, s)$ respectively, although $Q \times Q$ will mainly denote the domain of integration with respect to four variables. The following entropy error identity extends similar result in [4].

Lemma 4.3. Let $\phi=\phi(x, t ; y, s)$ be a nonnegative function such that

$$
\begin{aligned}
& (x, t) \mapsto \phi(x, t ; y, s) \in C_{c}^{\infty}(Q) \text { for every }(y, s) \in Q, \\
& (y, s) \mapsto \phi(x, t ; y, s) \in C_{c}^{\infty}(Q) \text { for every }(x, t) \in Q .
\end{aligned}
$$


Then we have

$$
\begin{aligned}
-\int_{Q \times Q} & U_{\varepsilon}\left(u, U_{h}\right)\left(\partial_{t} \phi+\partial_{s} \phi\right)-\int_{Q \times Q} F_{\varepsilon}\left(u, U_{h}\right)\left(\nabla_{x} \phi+\nabla_{y} \phi\right) \\
& +\int_{Q \times Q} H_{\varepsilon}\left(A(u)-A\left(U_{h}\right)\right) \nabla_{y} A(u) \cdot\left(\nabla_{x} \phi+\nabla_{y} \phi\right) \\
& +\int_{Q \times Q} H_{\varepsilon}\left(A\left(U_{h}\right)-A(u)\right) \nabla_{x} A\left(U_{h}\right) \cdot\left(\nabla_{x} \phi+\nabla_{y} \phi\right) \\
& +\int_{Q \times Q} H_{\varepsilon}^{\prime}\left(A(u)-A\left(U_{h}\right)\right)\left|\nabla_{x} A\left(U_{h}\right)-\nabla_{y} A(u)\right|^{2} \phi \\
= & -\int_{Q \times Q} \partial_{t}\left[U_{\varepsilon}\left(U_{h}, u\right)-U_{\varepsilon}\left(u, U_{h}\right)\right] \phi \\
& -\int_{Q \times Q} \nabla_{x}\left[F_{\varepsilon}\left(U_{h}, u\right)-F_{\varepsilon}\left(u, U_{h}\right)\right] \phi \\
& -\int_{Q(y, s)} \int_{0}^{T}\left\langle\mathcal{R}, H_{\varepsilon}\left(A\left(U_{h}\right)-A(u)\right) \phi\right\rangle d t .
\end{aligned}
$$

Proof. Recall that we write $u=u(y, s)$, and so we can take $k=U_{h}(x, t)$ in (4.1). Similarly, we can take $k^{\prime}=u(y, s)$ in (4.3). The lemma follows from the following two identities which can easily be proved by integration by parts:

$$
\begin{aligned}
& \int_{Q \times Q} H_{\varepsilon}\left(A(u)-A\left(U_{h}\right)\right) \nabla_{y} A(u) \cdot \nabla_{x} \phi \\
&= \int_{Q \times Q} H_{\varepsilon}^{\prime}\left(A(u)-A\left(U_{h}\right)\right) \nabla_{x} A\left(U_{h}\right) \cdot \nabla_{y} A(u) \phi, \\
& \int_{Q \times Q} H_{\varepsilon}\left(A\left(U_{h}\right)-A(u)\right) \nabla_{x} A\left(U_{h}\right) \cdot \nabla_{y} \phi \\
&=\int_{Q \times Q} H_{\varepsilon}^{\prime}\left(A\left(U_{h}\right)-A(u)\right) \nabla_{x} A\left(U_{h}\right) \cdot \nabla_{y} A(u) \phi .
\end{aligned}
$$

The next objective is to remove the restriction that the test functions in the entropy error identity (4.4) must have vanishing trace. This is achieved by using the technique of boundary layer sequence introduced in [27. The properties of the boundary layer sequence are summarized in the following lemma. For a proof, we refer to [27].

Lemma 4.4. For any $\delta>0$, let $\zeta_{\delta}$ be the solution of the elliptic problem

$$
-\delta^{2} \Delta \zeta_{\delta}+\zeta_{\delta}=1 \quad \text { in } \Omega, \quad \zeta_{\delta}=0 \quad \text { on } \partial \Omega
$$

Then we have

$$
\lim _{\delta \rightarrow 0} \zeta_{\delta}=1 \text { a.e. in } \Omega, \quad 0 \leq \zeta_{\delta} \leq 1, \quad-\Delta \zeta_{\delta} \geq 0 \text { in } \Omega
$$

Moreover, for any $v \in L^{2}\left(0, T ; H^{1}(\Omega)\right)$, and for any $\xi \in H^{1}(Q) \cap C(Q)$ such that $\xi(\cdot, 0)=\xi(\cdot, T)=0$,

$$
\lim _{\delta \rightarrow 0} \int_{Q}\left(v \cdot \nabla \zeta_{\delta}\right) \xi d x=-\int_{\Sigma}(v \cdot \nu) \xi
$$

where $\Sigma=\partial \Omega \times(0, T)$. 
Now we specify the choice of the test function $\phi$ in the entropy error identity (4.4), which is similar to that used in [27].

Definition 4.5. Let

$$
\phi(x, t, y, s)=\zeta_{\delta}(x) \zeta_{\eta}(y) \xi(x, t, y, s) \theta(t),
$$

where $\theta \in C_{c}^{\infty}(0, T)$ such that $\theta \geq 0$, and $\xi$ is defined as follows. Let $\left\{\varphi_{j}\right\}_{0 \leq j \leq J}$ be a partition of unity subordinate to open sets $B_{0}, B_{1}, \ldots, B_{J}$ such that $\bar{\Omega} \subset \bigcup_{j=0}^{J} B_{j}$, $B_{0} \subset \subset \Omega$ and $\partial \Omega \subset \bigcup_{j=1}^{J} B_{j}$. Let $\hat{\varphi}_{j} \in C_{c}^{\infty}\left(\mathbf{R}^{d}\right), 0 \leq \hat{\varphi}_{j} \leq 1$, such that $\operatorname{supp}\left(\hat{\varphi}_{j}\right) \subset$ $B_{j}$ and $\hat{\varphi}_{j}(x)=1$ on the support of $\varphi_{j}$ so that $\varphi_{j}(x) \hat{\varphi}_{j}(x)=\varphi_{j}(x)$. We use $\varphi_{j}$ as a function of $y$ and $\hat{\varphi}_{j}$ as a function of $x$, and denote $\hat{\varphi}_{j}(x) \varphi_{j}(y)=\psi_{j}(x, y)$. Define

$$
\xi(x, t, y, s)=\sum_{j=0}^{J} \omega_{l}(t-s) \omega_{m}\left(x^{\prime}-y^{\prime}\right) \omega_{n}\left(x_{d}-y_{d}\right) \psi_{j}(x, y),
$$

where $\omega_{l}, \omega_{n}$ are sequences of symmetric mollifiers in $\mathbf{R}, \omega_{m}$ is a sequence of symmetric mollifier in $\mathbf{R}^{d-1}$, and for $j=1,2, \ldots, J, x=\left(x^{\prime}, x_{d}\right), y=\left(y^{\prime}, y_{d}\right)$ are local coordinates induced by $\psi_{j}(x, y)$ in $B_{j}$. That is, $B_{j} \cap \partial \Omega=\left\{x \in B_{j}: x_{d}=\rho_{j}\left(x^{\prime}\right)\right\}$, $B \cap \Omega=\left\{x \in B_{j}: x_{d}<\rho_{j}\left(x^{\prime}\right)\right\}$ for some Lipschitz continuous function $\rho_{j}: \mathbf{R}^{d-1} \rightarrow$ R.

The following theorem is the main result of this section.

Theorem 4.6. Let $\theta$ and $\xi$ be defined as in Definition 4.5. Then we have the following entropy error inequality:

$$
\begin{aligned}
-\int_{Q \times Q} & U_{\varepsilon}\left(u, U_{h}\right) \xi \theta_{t}-\int_{Q \times Q} K_{\varepsilon}\left(u, U_{h}\right) \cdot\left(\nabla_{x} \xi+\nabla_{y} \xi\right) \theta \\
& +\int_{Q \times Q} H_{\varepsilon}^{\prime}\left(A(u)-A\left(U_{h}\right)\right)\left|\nabla_{x} A\left(U_{h}\right)-\nabla_{y} A(u)\right|^{2} \xi \theta \\
\leq & -\int_{Q \times Q} \partial_{t}\left[U_{\varepsilon}\left(U_{h}, u\right)-U_{\varepsilon}\left(u, U_{h}\right)\right] \xi \theta \\
& -\int_{Q \times Q} \nabla_{x}\left[F_{\varepsilon}\left(u, U_{h}\right)-F_{\varepsilon}\left(u, U_{h}\right)\right] \xi \theta \\
& -\int_{Q_{(y, s)}} \int_{\Sigma_{(x, t)}}\left(F_{\varepsilon}\left(u, U_{h}\right)-H_{\varepsilon}\left(A(u)-A\left(U_{h}\right)\right) \nabla_{y} A(u)\right) \cdot \nu_{x} \xi \theta \\
& -\int_{Q_{(x, t)}} \int_{\Sigma_{(y, s)}}\left(F_{\varepsilon}\left(u, U_{h}\right)-H_{\varepsilon}\left(A\left(U_{h}\right)-A(u)\right) \nabla_{x} A\left(U_{h}\right)\right) \cdot \nu_{y} \xi \theta \\
& -\int_{Q_{(y, s)}} \int_{0}^{T}\left\langle\mathcal{R}, H_{\varepsilon}\left(A\left(U_{h}\right)-A(u)\right) \xi \theta\right\rangle d t,
\end{aligned}
$$

where $K_{\varepsilon}\left(u, U_{h}\right)=F_{\varepsilon}\left(u, U_{h}\right)-H_{\varepsilon}\left(A(u)-A\left(U_{h}\right)\right)\left(\nabla_{y} A(u)-\nabla_{x} A\left(U_{h}\right)\right), \Sigma=\partial \Omega \times$ $(0, T)$, and $\Sigma_{(x, t)}$ or $\Sigma_{(y, s)}$ are the domain of integration of $\Sigma$ with respect to $(x, t)$ or $(y, s)$, respectively.

The proof of the theorem depends on the following lemmas.

Lemma 4.7. We have

$$
\lim _{\delta, \eta \rightarrow 0}-\int_{Q \times Q} U_{\varepsilon}\left(u, U_{h}\right)\left(\partial_{t} \phi+\partial_{s} \phi\right)=-\int_{Q \times Q} U_{\varepsilon}\left(u, U_{h}\right) \xi \theta_{t}
$$


and

$$
\begin{aligned}
\lim _{\delta, \eta \rightarrow 0}-\int_{Q \times Q} F_{\varepsilon}\left(u, U_{h}\right)\left(\nabla_{x} \phi+\nabla_{y} \phi\right)= & -\int_{Q \times Q} F_{\varepsilon}\left(u, U_{h}\right)\left(\nabla_{x} \xi+\nabla_{y} \xi\right) \theta \\
& +\int_{Q_{(y, s)}} \int_{\Sigma_{(x, t)}} F_{\varepsilon}\left(u, U_{h}\right) \cdot \nu_{x} \xi \theta \\
& +\int_{Q_{(x, t)}} \int_{\Sigma_{(y, s)}} F_{\varepsilon}\left(u, U_{h}\right) \cdot \nu_{y} \xi \theta
\end{aligned}
$$

Proof. By the definition of $\phi$ in (4.5) and $\xi$ in (4.6), we know that $\partial_{t} \phi+\partial_{s} \phi=$ $\zeta_{\delta} \zeta_{\eta} \xi \theta_{t}$. Thus

$$
\int_{Q \times Q} U_{\varepsilon}\left(u, U_{h}\right)\left(\partial_{t} \phi+\partial_{s} \phi\right)=\int_{Q \times Q} U_{\varepsilon}\left(u, U_{h}\right) \zeta_{\delta} \zeta_{\eta} \xi \theta_{t} .
$$

Then (4.8) follows by letting $\delta, \eta \rightarrow 0$ in the above equality and using the Lebesgue dominated convergence theorem.

Next, we note that

$$
\nabla_{x} \phi+\nabla_{y} \phi=\zeta_{\delta} \zeta_{\eta}\left(\nabla_{x} \xi+\nabla_{y} \xi\right) \theta+\left(\zeta_{\eta} \nabla_{x} \zeta_{\delta}+\zeta_{\delta} \nabla_{y} \zeta_{\eta}\right) \xi \theta
$$

Thus

$$
\begin{aligned}
\int_{Q \times Q} F_{\varepsilon}\left(u, U_{h}\right)\left(\nabla_{x} \phi+\nabla_{y} \phi\right)= & \int_{Q \times Q} F_{\varepsilon}\left(u, U_{h}\right) \zeta_{\delta} \zeta_{\eta}\left(\nabla_{x} \xi+\nabla_{y} \xi\right) \theta \\
& +\int_{Q \times Q} F_{\varepsilon}\left(u, U_{h}\right)\left(\zeta_{\eta} \nabla_{x} \zeta_{\delta}+\zeta_{\delta} \nabla_{y} \zeta_{\eta}\right) \xi \theta .
\end{aligned}
$$

Now we let $\delta, \eta \rightarrow 0$. The first term can be treated by using the Lebesgue dominated convergence theorem and the second term can be treated by Lemma 4.4 because of $F_{\varepsilon}\left(u, U_{h}\right) \in L^{2}\left(0, T ; H^{1}(\Omega)\right)$. This proves (4.9).

Lemma 4.8. We have

$$
\begin{aligned}
\varliminf_{\delta, \eta \rightarrow 0} & \int_{Q \times Q} H_{\varepsilon}\left(A(u)-A\left(U_{h}\right)\right) \nabla_{y} A(u) \cdot\left(\nabla_{x} \phi+\nabla_{y} \phi\right) \\
& \geq \int_{Q \times Q} H_{\varepsilon}\left(A(u)-A\left(U_{h}\right)\right) \nabla_{y} A(u) \cdot\left(\nabla_{x} \xi+\nabla_{y} \xi\right) \theta \\
& -\int_{Q_{(y, s)}} \int_{\Sigma_{(x, t)}} H_{\varepsilon}\left(A(u)-A\left(U_{h}\right)\right) \nabla_{y} A(u) \cdot \nu_{x} \xi \theta .
\end{aligned}
$$

Proof. By (4.9) we have

$$
\begin{aligned}
\int_{Q \times Q} & H_{\varepsilon}\left(A(u)-A\left(U_{h}\right)\right) \nabla_{y} A(u) \cdot\left(\nabla_{x} \phi+\nabla_{y} \phi\right) \\
= & \int_{Q \times Q} H_{\varepsilon}\left(A(u)-A\left(U_{h}\right)\right) \nabla_{y} A(u) \cdot\left(\nabla_{x} \xi+\nabla_{y} \xi\right) \zeta_{\delta} \zeta_{\eta} \theta \\
& +\int_{Q \times Q} H_{\varepsilon}\left(A(u)-A\left(U_{h}\right)\right) \nabla_{y} A(u) \cdot \nabla_{x} \zeta_{\delta} \zeta_{\eta} \xi \theta \\
& +\int_{Q \times Q} H_{\varepsilon}\left(A(u)-A\left(U_{h}\right)\right) \nabla_{y} A(u) \cdot \nabla_{y} \zeta_{\eta} \zeta_{\delta} \xi \theta
\end{aligned}
$$


By the Lebesgue dominated convergence theorem, we get

$$
\begin{gathered}
\lim _{\delta, \eta \rightarrow 0} \int_{Q \times Q} H_{\varepsilon}\left(A(u)-A\left(U_{h}\right)\right) \nabla_{y} A(u) \cdot\left(\nabla_{x} \xi+\nabla_{y} \xi\right) \zeta_{\delta} \zeta_{\eta} \theta \\
=\int_{Q \times Q} H_{\varepsilon}\left(A(u)-A\left(U_{h}\right)\right) \nabla_{y} A(u) \cdot\left(\nabla_{x} \xi+\nabla_{y} \xi\right) \theta .
\end{gathered}
$$

By Lemma 4.4 and the Lebesgue dominated convergence theorem, we have

$$
\begin{aligned}
& \lim _{\delta, \eta \rightarrow 0} \int_{Q \times Q} H_{\varepsilon}\left(A(u)-A\left(U_{h}\right)\right) \nabla_{y} A(u) \cdot \nabla_{x} \zeta_{\delta} \zeta_{\eta} \xi \theta \\
& =-\int_{Q_{(y, s)}} \int_{\Sigma_{(x, t)}} H_{\varepsilon}\left(A(u)-A\left(U_{h}\right)\right) \nabla_{y} A(u) \cdot \nu_{x} \xi \theta .
\end{aligned}
$$

To deal with the third term on the right-hand side of (4.10), we define

$$
\Phi_{\varepsilon}(z)=\int_{0}^{z} H_{\varepsilon}(r) d r, \quad \forall z \in \mathbf{R} .
$$

It is easy to see that $\Phi_{\varepsilon}(z)=\Phi_{\varepsilon}(-z)$ and $\Phi_{\varepsilon}\left(z_{1}+z_{2}\right) \leq \Phi_{\varepsilon}\left(z_{1}\right)+\Phi_{\varepsilon}\left(z_{2}\right)$. Thus

$\Psi_{\varepsilon}\left(u, U_{h}\right)=\Phi_{\varepsilon}\left(A(u)-A\left(U_{h}\right)\right)+\Phi_{\varepsilon}(A(u))-\Phi_{\varepsilon}\left(A\left(U_{h}\right)\right) \geq 0 \quad$ a.e. in $Q \times Q$.

Note that

$$
\begin{aligned}
\int_{Q \times Q} & H_{\varepsilon}\left(A(u)-A\left(U_{h}\right)\right) \nabla_{y} A(u) \cdot \nabla_{y} \zeta_{\eta} \zeta_{\delta} \xi \theta \\
= & \int_{Q \times Q} \nabla_{y} \Phi_{\varepsilon}\left(A(u)-A\left(U_{h}\right)\right) \cdot \nabla_{y} \zeta_{\eta} \zeta_{\delta} \xi \theta \\
= & \int_{Q \times Q} \nabla_{y}\left[\Phi_{\varepsilon}\left(A(u)-A\left(U_{h}\right)\right)-\Phi_{\varepsilon}\left(A\left(U_{h}\right)\right)\right] \cdot \nabla_{y} \zeta_{\eta} \zeta_{\delta} \xi \theta \\
= & \int_{Q \times Q} \nabla_{y} \Psi_{\varepsilon}\left(u, U_{h}\right) \cdot \nabla_{y} \zeta_{\eta} \zeta_{\delta} \xi \theta-\int_{Q \times Q} \nabla_{y} \Phi_{\varepsilon}(A(u)) \cdot \nabla_{y} \zeta_{\eta} \zeta_{\delta} \xi \theta \\
= & : L_{1}+L_{2} .
\end{aligned}
$$

Since $\Psi_{\varepsilon}\left(u, U_{h}\right) \geq 0,-\Delta_{y} \zeta_{\eta} \geq 0$, by integrating by parts we get

$$
\begin{aligned}
L_{1} & =-\int_{Q \times Q} \Psi_{\varepsilon}\left(u, U_{h}\right) \Delta_{y} \zeta_{\eta} \zeta_{\delta} \xi \theta-\int_{Q \times Q} \Psi_{\varepsilon}\left(u, U_{h}\right) \nabla_{y} \zeta_{\eta} \cdot \nabla_{y} \xi \zeta_{\delta} \theta \\
& \geq-\int_{Q \times Q} \Psi_{\varepsilon}\left(u, U_{h}\right) \nabla_{y} \zeta_{\eta} \cdot \nabla_{y} \xi \zeta_{\delta} \theta .
\end{aligned}
$$

Since $\left.\Psi_{\varepsilon}\left(u, U_{h}\right)\right|_{\Sigma_{(y, s)}}=0$, we deduce by using Lemma 4.4 that

$$
\varliminf_{\delta, \eta \rightarrow 0} L_{1} \geq 0
$$


Note that, by using Lebesgue dominated convergence theorem, we have

$$
\begin{aligned}
\lim _{\delta \rightarrow 0} L_{2}= & -\int_{Q \times Q} H_{\varepsilon}(A(u)) \nabla_{y} A(u) \cdot \nabla_{y} \zeta_{\eta} \xi \theta \\
= & \int_{Q \times Q} H_{\varepsilon}(A(u)) \nabla_{y} A(u) \cdot \nabla_{y}\left[\left(1-\zeta_{\eta}\right) \xi \theta\right] \\
& -\int_{Q \times Q} H_{\varepsilon}(A(u)) \nabla_{y} A(u) \cdot \nabla_{y}(\xi \theta)\left(1-\zeta_{\eta}\right) \\
=: & L_{21}+L_{22} .
\end{aligned}
$$

Since $\zeta_{\eta} \rightarrow 1$ a.e. in $\Omega$, we obtain

$$
\lim _{\eta \rightarrow 0} L_{22}=0 .
$$

To deal with $L_{21}$, we denote it by

$$
\Theta(y, s)=\left(1-\zeta_{\eta}(y)\right) \int_{Q} \xi(x, t, y, s) \theta(t) d x d t .
$$

Since $\theta \in C_{c}^{\infty}(0, T)$, for sufficiently large $l$, we have that $\Theta(\cdot, 0)=\Theta(\cdot, T)=0$. Thus we can take $\varphi=H_{\varepsilon}(A(u)) \Theta$ as the test function in (2.4). Using a similar argument leading to (4.1), we can show that

$$
\begin{gathered}
-\int_{Q} U_{\varepsilon}(u, 0) \partial_{s} \Theta-\int_{Q} F_{\varepsilon}(u, 0) \cdot \nabla_{y} \Theta+\int_{Q} H_{\varepsilon}(A(u)) \nabla_{y} A(u) \cdot \nabla_{y} \Theta \\
+\int_{Q} H_{\varepsilon}^{\prime}(A(u))\left|\nabla_{y} A(u)\right|^{2} \Theta=\int_{Q} g H_{\varepsilon}(A(u)) \Theta .
\end{gathered}
$$

Therefore

$$
\begin{aligned}
L_{21}= & \int_{Q} g H_{\varepsilon}(A(u)) \Theta+\int_{Q} U_{\varepsilon}(u, 0) \partial_{s} \Theta-\int_{Q} \operatorname{div}_{y} F_{\varepsilon}(u, 0) \Theta \\
& -\int_{Q} H_{\varepsilon}^{\prime}(A(u))\left|\nabla_{y} A(u)\right|^{2} \Theta,
\end{aligned}
$$

which tends to zero as $\eta \rightarrow 0$ by using the fact that $\Theta, \partial_{s} \Theta \rightarrow 0$ as $\eta \rightarrow 0$. This proves that $\lim _{\eta \rightarrow 0} \lim _{\delta \rightarrow 0} L_{2}=0$. Similarly, we can show that $\lim _{\delta \rightarrow 0} \lim _{\eta \rightarrow 0} L_{2}=$ 0 . This completes the proof.

Now we are ready to prove Theorem 4.6 .

Proof of Theorem 4.6. The proof lies in taking the test function $\phi$ in the entropy error identity (4.4) as in Definition 4.5, and then taking the limit $\delta, \eta \rightarrow 0$. By Lemmas 4.7 and 4.8 and the Lebesgue dominated convergence theorem, it remains only to consider the limit of the following quantity:

$$
\begin{aligned}
L_{3}= & \int_{Q \times Q} H_{\varepsilon}\left(A\left(U_{h}\right)-A(u)\right) \nabla_{x} A\left(U_{h}\right) \cdot\left(\nabla_{x} \phi+\nabla_{y} \phi\right) \\
& +\int_{Q_{(y, s)}} \int_{0}^{T}\left\langle\mathcal{R}, H_{\varepsilon}\left(A\left(U_{h}\right)-A(u)\right) \phi\right\rangle d t .
\end{aligned}
$$


From (4.9) and (4.2), it is easy to check that

$$
\begin{aligned}
L_{3}=\int_{Q \times Q} & H_{\varepsilon}\left(A\left(U_{h}\right)-A(u)\right) \nabla_{x} A\left(U_{h}\right) \cdot\left(\nabla_{x} \xi+\nabla_{y} \xi\right) \zeta_{\delta} \zeta_{\eta} \theta \\
& +\int_{Q \times Q} H_{\varepsilon}\left(A\left(U_{h}\right)-A(u)\right) \nabla_{x} A\left(U_{h}\right) \cdot \nabla_{y} \zeta_{\eta} \zeta_{\delta} \xi \theta \\
& +\int_{Q_{(y, s)}} \int_{0}^{T}\left\langle g-\partial_{t} U_{h}, H_{\varepsilon}\left(A\left(U_{h}\right)-A(u)\right) \zeta_{\delta} \zeta_{\eta} \xi \theta\right\rangle d t \\
& +\int_{Q_{(y, s)}} \int_{0}^{T}\left\langle f\left(U_{h}\right), \nabla_{x}\left(H_{\varepsilon}\left(A\left(U_{h}\right)-A(u)\right) \xi \theta\right) \zeta_{\delta} \zeta_{\eta}\right\rangle d t \\
& +\int_{Q_{(y, s)}} \int_{0}^{T}\left\langle f\left(U_{h}\right), \nabla_{x} \zeta_{\delta} \zeta_{\eta} H_{\varepsilon}\left(A\left(U_{h}\right)-A(u)\right) \xi \theta\right\rangle d t \\
& -\int_{Q_{(y, s)}} \int_{0}^{T}\left\langle\nabla_{x} A\left(U_{h}\right), \nabla_{x}\left(H_{\varepsilon}\left(A\left(U_{h}\right)-A(u)\right) \xi \theta\right) \zeta_{\delta} \zeta_{\eta}\right\rangle d t \\
=: \quad L_{31} & +\cdots+L_{36} .
\end{aligned}
$$

By the Lebesgue dominated convergence theorem, we have

$$
\lim _{\delta, \eta \rightarrow 0} L_{31}=\int_{Q \times Q} H_{\varepsilon}\left(A\left(U_{h}\right)-A(u)\right) \nabla_{x} A\left(U_{h}\right) \cdot\left(\nabla_{x} \xi+\nabla_{y} \xi\right) \theta
$$

and

$$
\lim _{\delta, \eta \rightarrow 0}\left(L_{33}+L_{34}+L_{36}\right)=\int_{Q_{(y, s)}} \int_{0}^{T}\left\langle\mathcal{R}, H_{\varepsilon}\left(A\left(U_{h}\right)-A(u)\right) \xi \theta\right\rangle d t .
$$

By Lemma 4.4, we get

$$
\lim _{\delta, \eta \rightarrow 0} L_{32}=-\int_{Q_{(x, t)}} \int_{\Sigma_{(y, s)}} H_{\varepsilon}\left(A\left(U_{h}\right)-A(u)\right) \nabla_{x} A\left(U_{h}\right) \cdot \nu_{y} \xi \theta .
$$

Moreover, since $f\left(U_{h}\right)=0$ on $\Sigma_{(x, t)}$, we have

$$
\lim _{\delta, \eta \rightarrow 0} L_{35}=0 .
$$

Therefore we have

$$
\begin{aligned}
\lim _{\delta, \eta \rightarrow 0} L_{3}= & \int_{Q \times Q} H_{\varepsilon}\left(A\left(U_{h}\right)-A(u)\right) \nabla_{x} A\left(U_{h}\right) \cdot\left(\nabla_{x} \xi+\nabla_{y} \xi\right) \theta \\
& -\int_{Q_{(x, t)}} \int_{\Sigma_{(y, s)}} H_{\varepsilon}\left(A\left(U_{h}\right)-A(u)\right) \nabla_{x} A\left(U_{h}\right) \cdot \nu_{y} \xi \theta \\
& +\int_{Q_{(y, s)}} \int_{0}^{T}\left\langle\mathcal{R}, H_{\varepsilon}\left(A\left(U_{h}\right)-A(u)\right) \xi \theta\right\rangle d t
\end{aligned}
$$

This completes the proof.

5. A POSTERIORI ERROR ANALYSIS

For any $\varepsilon>0$ and $z \in \mathbf{R}$, define

$$
\nu(\varepsilon, z)=\min \left\{A^{\prime}(s):|A(s)-A(z)| \leq \varepsilon\right\} .
$$

We start by prove the following elementary estimate which extends the result in [12, Corollary 6.4]. 
Lemma 5.1. For any $k \in \mathbf{R}$ and $z \in \mathbf{R}$, we have

$$
\begin{aligned}
& \left|\partial_{z}\left[U_{\varepsilon}(z, k)-U_{\varepsilon}(k, z)\right]\right| \leq \frac{\varepsilon^{\gamma}}{\nu(\varepsilon, z)} \mathcal{K}_{1}, \\
& \left|\partial_{z}\left[F_{\varepsilon}(z, k)-F_{\varepsilon}(k, z)\right]\right| \leq \frac{\varepsilon^{\gamma}}{\nu(\varepsilon, z)} \mathcal{K}_{2},
\end{aligned}
$$

where $0<\gamma \leq 1$ is the Hölder exponent of $A^{\prime} \circ A^{-1}$ in $(\mathrm{H} 2), \mathcal{K}_{1}=\mathrm{H}\left(A^{\prime} \circ A^{-1}\right)$, $\mathcal{K}_{2}=\mathcal{K}_{1}\left\|f^{\prime}\right\|_{L^{\infty}(\mathbf{R})}+\mathrm{L}\left(f^{\prime}\right)$ with $\mathrm{H}\left(A^{\prime} \circ A^{-1}\right)$ and $\mathrm{L}\left(f^{\prime}\right)$ being the Hölder constant of $A^{\prime} \circ A^{-1}$ and the Lipschitz constant of $f^{\prime}$ respectively.

Proof. We only prove the estimate for $F_{\varepsilon}$. The estimate for $U_{\varepsilon}$ is similar. By definition,

$$
\begin{aligned}
& \partial_{z}\left[F_{\varepsilon}(z, k)-F_{\varepsilon}(k, z)\right] \\
= & \partial_{z} \int_{k}^{z}\left[H_{\varepsilon}(A(r)-A(k))+H_{\varepsilon}(A(r)-A(z))\right] f^{\prime}(r) d r \\
= & H_{\varepsilon}(A(z)-A(k)) f^{\prime}(z)-\int_{k}^{z} H_{\varepsilon}^{\prime}(A(r)-A(z)) A^{\prime}(z) f^{\prime}(r) d r \\
= & \int_{k}^{z} H_{\varepsilon}^{\prime}(A(r)-A(z)) A^{\prime}(r) f^{\prime}(z) d r-\int_{k}^{z} H_{\varepsilon}^{\prime}(A(r)-A(z)) A^{\prime}(z) f^{\prime}(r) d r \\
= & \int_{k}^{z} H_{\varepsilon}^{\prime}(A(r)-A(z)) A^{\prime}(r)\left(f^{\prime}(z)-f^{\prime}(r)\right) d r \\
& +\int_{k}^{z} H_{\varepsilon}^{\prime}(A(r)-A(z))\left(A^{\prime}(r)-A^{\prime}(z)\right) f^{\prime}(r) d r .
\end{aligned}
$$

Without loss of generality, we may assume $z>k$. Then since $H_{\varepsilon}^{\prime}(A(r)-A(z))$ vanishes outside the set $\{s:|A(s)-A(z)| \leq \varepsilon\}$, we know that

$$
\begin{aligned}
& \left|\int_{k}^{z} H_{\varepsilon}^{\prime}(A(r)-A(z)) A^{\prime}(r)\left(f^{\prime}(z)-f^{\prime}(r)\right) d r\right| \\
\leq & \frac{1}{\varepsilon} \int_{A^{-1}(A(z)-\varepsilon)}^{z} A^{\prime}(r)\left|f^{\prime}(r)-f^{\prime}(z)\right| d r \\
\leq & \frac{1}{\varepsilon} \mathrm{L}\left(f^{\prime}\right)\left|z-A^{-1}(A(z)-\varepsilon)\right| \int_{A^{-1}(A(z)-\varepsilon)}^{z} A^{\prime}(r) d r \\
\leq & \mathrm{L}\left(f^{\prime}\right) \frac{\varepsilon}{\nu(\varepsilon, z)},
\end{aligned}
$$

where we have used the fact that $z=A^{-1}(A(z))$. Moreover,

$$
\begin{aligned}
& \left|\int_{k}^{z} H_{\varepsilon}^{\prime}(A(r)-A(z))\left(A^{\prime}(r)-A^{\prime}(z)\right) f^{\prime}(r) d r\right| \\
\leq & \mathrm{H}\left(A^{\prime} \circ A^{-1}\right) \varepsilon^{\gamma-1} \int_{A^{-1}(A(z)-\varepsilon)}^{z}\left|f^{\prime}(r)\right| d r \\
\leq & \mathrm{H}\left(A^{\prime} \circ A^{-1}\right) \varepsilon^{\gamma-1}\left\|f^{\prime}\right\|_{L^{\infty}(\mathbf{R})}\left|z-A^{-1}(A(z)-\varepsilon)\right| \\
\leq & \mathrm{H}\left(A^{\prime} \circ A^{-1}\right)\left\|f^{\prime}\right\|_{L^{\infty}(\mathbf{R})} \frac{\varepsilon^{\gamma}}{\nu(\varepsilon, z)} .
\end{aligned}
$$

This completes the proof. 
The next step is to let the parameters in the mollifier functions $l, m, n \rightarrow \infty$ in the entropy error inequality (4.7) and definitions (4.5)-(4.6) complete the Kružkov "doubling of variables" technique.

Lemma 5.2. We have

$$
\begin{gathered}
\lim _{l, m, n \rightarrow \infty} \int_{Q \times Q} U_{\varepsilon}\left(u, U_{h}\right) \xi \theta_{t}=\int_{Q} U_{\varepsilon}\left(u, U_{h}\right) \theta_{t}, \\
\lim _{l, m, n \rightarrow \infty} \int_{Q \times Q} K_{\varepsilon}\left(u, U_{h}\right) \cdot\left(\nabla_{x} \xi+\nabla_{y} \xi\right) \theta=0, \\
\lim _{l, m, n \rightarrow \infty} \int_{Q \times Q} H_{\varepsilon}^{\prime}\left(A(u)-A\left(U_{h}\right)\right)\left|\nabla_{x} A\left(U_{h}\right)-\nabla_{y} A(u)\right|^{2} \xi \theta \\
\quad=\int_{Q} H_{\varepsilon}^{\prime}\left(A(u)-A\left(U_{h}\right)\right)\left|\nabla A\left(U_{h}\right)-\nabla A(u)\right|^{2} \theta .
\end{gathered}
$$

Proof. From the definition of $\xi(x, t, y, s)$ in (4.6) and the properties of mollifier functions, we have

$$
\lim _{l, m, n \rightarrow \infty} \int_{Q \times Q} U_{\varepsilon}\left(u, U_{h}\right) \xi \theta_{t}=\sum_{j=0}^{J} \int_{Q} U_{\varepsilon}\left(u, U_{h}\right) \psi_{j}(x, x) \theta_{t}=\int_{Q} U_{\varepsilon}\left(u, U_{h}\right) \theta_{t},
$$

where we have used the fact that

$$
\sum_{j=0}^{J} \psi_{j}(x, x)=\sum_{j=0}^{J} \varphi_{j}(x) \hat{\varphi}_{j}(x)=\sum_{j=0}^{J} \varphi_{j}(x)=1 \quad \text { for } x \in \Omega .
$$

This proves (5.2). Similarly, we can show (5.4). To see (5.3), we note that

$$
\nabla_{x} \xi+\nabla_{y} \xi=\sum_{j=0}^{J} \omega_{l}(t-s) \omega_{m}\left(x^{\prime}-y^{\prime}\right) \omega_{n}\left(x_{d}-y_{d}\right)\left(\nabla_{x} \psi_{j}+\nabla_{y} \psi_{j}\right)
$$

Thus

$$
\lim _{l, m, n \rightarrow \infty} \int_{Q \times Q} K_{\varepsilon}\left(u, U_{h}\right) \cdot\left(\nabla_{x} \xi+\nabla_{y} \xi\right) \theta=\sum_{j=0}^{J} \int_{Q} K_{\varepsilon}\left(u, U_{h}\right) \nabla_{x} \psi_{j}(x, x) \theta,
$$

which vanishes due to (5.5).

Lemma 5.3. We have

$$
\begin{aligned}
& \varlimsup_{l, m, n \rightarrow \infty}\left|\int_{Q \times Q} \partial_{t}\left(U_{\varepsilon}\left(U_{h}, u\right)-U_{\varepsilon}\left(u, U_{h}\right)\right) \xi \theta\right| \leq \mathcal{K}_{1} \varepsilon^{\gamma} \int_{Q} \frac{1}{\nu\left(\varepsilon, U_{h}\right)}\left|\partial_{t} U_{h}\right| \theta, \\
& \varlimsup_{l, m, n \rightarrow \infty}\left|\int_{Q \times Q} \nabla_{x}\left(F_{\varepsilon}\left(U_{h}, u\right)-F_{\varepsilon}\left(u, U_{h}\right)\right) \xi \theta\right| \leq \mathcal{K}_{2} \varepsilon^{\gamma} \int_{Q} \frac{1}{\nu\left(\varepsilon, U_{h}\right)}\left|\nabla_{x} U_{h}\right| \theta .
\end{aligned}
$$

Proof. By Lemma 5.1, we have

$$
\begin{aligned}
& \left|\int_{Q \times Q} \partial_{t}\left(U_{\varepsilon}\left(U_{h}, u\right)-U_{\varepsilon}\left(u, U_{h}\right)\right) \xi \theta\right| \\
\leq & \mathcal{K}_{1} \varepsilon^{\gamma} \int_{Q_{(x, t)}} \frac{1}{\nu\left(\varepsilon, U_{h}\right)}\left|\partial_{t} U_{h}\right| \theta \cdot \int_{Q_{(y, s)}} \xi d y d s .
\end{aligned}
$$


From the definition of $\xi$ in (4.6) and (5.5), it is easy to see that

$$
\lim _{l, m, n \rightarrow \infty} \int_{Q_{(y, s)}} \xi(x, t, y, s)=1 .
$$

This proves the estimate (5.6). Similarly we can show (5.7).

Lemma 5.4. We have

(5.8) $\lim _{n \rightarrow \infty} \lim _{l, m \rightarrow \infty} \int_{Q_{(y, s)}} \int_{\Sigma_{(x, t)}}\left(F_{\varepsilon}\left(u, U_{h}\right)-H_{\varepsilon}\left(A(u)-A\left(U_{h}\right)\right) \nabla_{y} A(u)\right) \cdot \nu_{x} \xi \theta=0$,

(5.9) $\lim _{n \rightarrow \infty} \lim _{l, m \rightarrow \infty} \int_{Q_{(x, t)}} \int_{\Sigma_{(y, s)}}\left(F_{\varepsilon}\left(u, U_{h}\right)-H_{\varepsilon}\left(A\left(U_{h}\right)-A(u)\right) \nabla_{x} A\left(U_{h}\right)\right) \cdot \nu_{y} \xi \theta=0$.

Proof. We modify the idea in [27, §3] to show (5.8). Since $U_{h}=0, A\left(U_{h}\right)=0$ on $\Sigma_{(x, t)}$, defining $N_{j}\left(y^{\prime}\right)=\left(-\nabla \rho_{j}\left(y^{\prime}\right), 1\right)$ (see Definition 4.5 for the notation) we get

$$
\begin{aligned}
& \lim _{l, m \rightarrow \infty} \int_{Q_{(y, s)}} \int_{\Sigma_{(x, t)}}\left(F_{\varepsilon}\left(u, U_{h}\right)-H_{\varepsilon}\left(A(u)-A\left(U_{h}\right)\right) \nabla_{y} A(u)\right) \cdot \nu_{x} \xi \theta \\
= & \sum_{j=1}^{J} \int_{Q_{(y, s)}}\left(F_{\varepsilon}(u, 0)-H_{\varepsilon}(A(u)) \nabla_{y} A(u)\right) \cdot N_{j}\left(y^{\prime}\right) \omega_{n}\left(\rho_{j}\left(y^{\prime}\right)-y_{d}\right) \psi_{j} \theta \\
= & : L_{4},
\end{aligned}
$$

where $\psi_{j}(y)=\hat{\varphi}_{j}\left(y^{\prime}, \rho_{j}\left(y^{\prime}\right)\right) \varphi_{j}\left(y^{\prime}, y_{d}\right)$. Let $w_{n}(y)=2 \int_{y_{d}-\rho_{j}\left(y^{\prime}\right)}^{0} \omega_{n}(s) d s$, then $\nabla w_{n}=-2 \omega_{n}\left(\rho_{j}\left(y^{\prime}\right)-y_{d}\right) N_{j}\left(y^{\prime}\right)$. Thus

$$
\begin{aligned}
L_{4}= & -\frac{1}{2} \sum_{j=1}^{J} \int_{Q_{(y, s)}}\left(F_{\varepsilon}(u, 0)-H_{\varepsilon}(A(u)) \nabla_{y} A(u)\right) \cdot \nabla w_{n} \psi_{j} \theta \\
= & \frac{1}{2} \sum_{j=1}^{J} \int_{Q_{(y, s)}}\left(F_{\varepsilon}(u, 0)-H_{\varepsilon}(A(u)) \nabla_{y} A(u)\right) \cdot \nabla\left[\left(1-w_{n}\right) \psi_{j} \theta\right] \\
& -\frac{1}{2} \sum_{j=1}^{J} \int_{Q_{(y, s)}}\left(F_{\varepsilon}(u, 0)-H_{\varepsilon}(A(u)) \nabla_{y} A(u)\right) \cdot \nabla\left(\psi_{j} \theta\right)\left(1-w_{n}\right) \\
= & : \quad L_{41}+L_{42} .
\end{aligned}
$$

Note that $w_{n} \rightarrow 1$ a.e. in $\Omega$. By the Lebesgue dominated convergence theorem, we have $\lim _{n \rightarrow \infty} L_{42}=0$. Moreover, by (4.11) and using the argument in dealing with the limit $L_{21}$ in the proof of Lemma 4.8, we can show that $\lim _{n \rightarrow \infty} L_{41}=0$. Therefore, $L_{4}$ tends to 0 as $n \rightarrow \infty$. This proves (5.8).

The proof of (5.9) is simpler. Since $U_{h}$ is a finite element function, the trace of $F_{\varepsilon}\left(U_{h}, 0\right)+F_{\varepsilon}\left(A\left(U_{h}\right)\right) \cdot \nabla_{x} A\left(U_{h}\right)$ on $\Sigma_{(x, t)}$ is well defined and is equal to 0 . One can easily prove the integral in (5.9) converges to zero as $l, m, n \rightarrow \infty$.

Lemma 5.5. Let $\theta$ be defined in Definition 4.5. Then we have

$$
\begin{aligned}
& -\int_{Q} U_{\varepsilon}\left(u, U_{h}\right) \theta_{t}+\int_{Q} H_{\varepsilon}^{\prime}\left(A(u)-A\left(U_{h}\right)\right)\left|\nabla\left(A\left(U_{h}\right)-A(u)\right)\right|^{2} \theta \\
\leq & \mathcal{K} \varepsilon^{\gamma} \int_{Q} \frac{1}{\nu\left(\varepsilon, U_{h}\right)}\left(\left|\partial_{t} U_{h}\right|+\left|\nabla_{x} U_{h}\right|\right) \theta-\int_{0}^{T}\left\langle\mathcal{R}, H_{\varepsilon}\left(A\left(U_{h}\right)-A(u)\right) \theta\right\rangle d t,
\end{aligned}
$$

where $\mathcal{K}=\max \left(\mathcal{K}_{1}, \mathcal{K}_{2}\right)$. 
Proof. We first let $l, m \rightarrow \infty$, then $n \rightarrow \infty$ in the entropy error inequality (4.7). By Lemmas 5.2 5.4, it remains to consider the limit of

$$
\begin{aligned}
L_{5}:= & -\int_{Q_{(y, s)}} \int_{0}^{T}\left\langle\mathcal{R}, H_{\varepsilon}\left(A\left(U_{h}\right)-A(u)\right) \xi \theta\right\rangle d t \\
= & -\int_{Q \times Q}\left(g-\partial_{t} U_{h}\right) H_{\varepsilon}\left(A\left(U_{h}\right)-A(u)\right) \xi \theta \\
& -\int_{Q \times Q}\left(f\left(U_{h}\right)-\nabla_{x} A\left(U_{h}\right)\right) \cdot \nabla_{x}\left(H_{\varepsilon}\left(A\left(U_{h}\right)-A(u)\right)\right) \xi \theta \\
& -\int_{Q \times Q}\left(f\left(U_{h}\right)-\nabla_{x} A\left(U_{h}\right)\right) \cdot \nabla_{x} \xi H_{\varepsilon}\left(A\left(U_{h}\right)-A(u)\right) \theta .
\end{aligned}
$$

Note that, by integration by parts, we have

$$
\begin{aligned}
& \int_{Q \times Q}\left(f\left(U_{h}\right)-\nabla_{x} A\left(U_{h}\right)\right) \cdot \nabla_{y} \xi H_{\varepsilon}\left(A\left(U_{h}\right)-A(u)\right) \theta \\
= & -\int_{Q \times Q}\left(f\left(U_{h}\right)-\nabla_{x} A\left(U_{h}\right)\right) \cdot \nabla_{y}\left(H_{\varepsilon}\left(A\left(U_{h}\right)-A(u)\right)\right) \xi \theta \\
& +\int_{Q_{(x, t)}} \int_{\Sigma_{(y, s)}} H_{\varepsilon}\left(A\left(U_{h}\right)\right)\left(f\left(U_{h}\right)-\nabla_{x} A\left(U_{h}\right)\right) \cdot \nu_{y} \xi \theta .
\end{aligned}
$$

Thus

$$
\begin{aligned}
L_{5}= & -\int_{Q \times Q}\left(g-\partial_{t} U_{h}\right) H_{\varepsilon}\left(A\left(U_{h}\right)-A(u)\right) \xi \theta \\
& -\int_{Q \times Q} H_{\varepsilon}^{\prime}\left(A\left(U_{h}\right)-A(u)\right)\left(f\left(U_{h}\right)-\nabla_{x} A\left(U_{h}\right)\right) \cdot\left(\nabla_{x} A\left(U_{h}\right)-\nabla_{y} A(u)\right) \xi \theta \\
& -\int_{Q \times Q}\left(f\left(U_{h}\right)-\nabla_{x} A\left(U_{h}\right)\right) \cdot\left(\nabla_{x} \xi+\nabla_{y} \xi\right) H_{\varepsilon}\left(A\left(U_{h}\right)-A(u)\right) \theta \\
& +\int_{Q_{(x, t)}} \int_{\Sigma_{(y, s)}} H_{\varepsilon}\left(A\left(U_{h}\right)\right)\left(f\left(U_{h}\right)-\nabla_{x} A\left(U_{h}\right)\right) \cdot \nu_{y} \xi \theta \\
=: & L_{51}+\cdots+L_{54} .
\end{aligned}
$$

Similar to the proof of $(5.2)$ in Lemma 5.2 , it is easy to see that

$$
\lim _{l, m, n \rightarrow \infty}\left(L_{51}+L_{52}\right)=-\int_{0}^{T}\left\langle\mathcal{R}, H_{\mathcal{\varepsilon}}\left(A\left(U_{h}\right)-A(u)\right) \theta\right\rangle d t .
$$

Similar to the proof of (5.3) in Lemma 5.2, we know that $\lim _{l, m, n \rightarrow \infty} L_{53}=0$. Finally, since $H_{\varepsilon}\left(A\left(U_{h}\right)\right)=0$ on $\Sigma_{(x, t)}$, we can easily prove that $L_{54} \rightarrow 0$ as $l, m, n \rightarrow \infty$. This completes the proof.

To proceed, we introduce the interior residual

$$
R^{n}:=\bar{g}^{n}-\frac{U_{h}^{n}-\bar{U}_{h}^{n-1}}{\tau_{n}}+\Delta A\left(U_{h}^{n}\right) \quad \text { on any } K \in \mathcal{M}^{n}
$$

where we recall that $\bar{g}^{n}=\tau_{n}^{-1} \int_{t^{n-1}}^{t^{n}} g(x, t) d t$.

The following theorem is the main result of this paper. 
Theorem 5.6. Let the assumptions $(\mathrm{H} 1)-(\mathrm{H} 3)$ be satisfied. Let $\varepsilon_{0}=\left(\sum_{i=1}^{3} \mathcal{E}_{i}\right)^{\frac{2}{1+\gamma}}$, where $\gamma$ is the Hölder exponent of $A^{\prime} \circ A^{-1}$, and $\mathcal{E}_{1}, \mathcal{E}_{2}, \mathcal{E}_{3}$ are the error indicators defined below. For any $m \geq 1$, let $Q_{m}=\Omega \times\left(0, t^{m}\right)$, and define

$$
\Lambda_{m}=\max \left(1, \int_{Q_{m}} \frac{1}{\nu\left(\varepsilon_{0}, U_{h}\right)}\left(\left|\partial_{t} U_{h}\right|+\left|\nabla U_{h}\right|\right)+\int_{\Omega} \frac{1}{\nu\left(\varepsilon_{0}, U_{h}^{m}\right)}\right)
$$

where for any $z \in \mathbf{R}, \nu\left(\varepsilon_{0}, z\right)=\min \left\{A^{\prime}(s):|A(s)-A(z)| \leq \varepsilon_{0}\right\}$. Then there exists a constant $C$ depending only on the minimum angles of the meshes $\mathcal{M}^{n}$, $n=1, \ldots, m$, such that the following a posteriori error estimate is valid:

$$
\left\|u^{m}-U_{h}^{m}\right\|_{L^{1}(\Omega)} \leq \mathcal{E}_{0}+\mathcal{E}_{4}+\mathcal{E}_{5}+C \Lambda_{m}^{\frac{1}{1+\gamma}}\left(\sum_{i=1}^{3} \mathcal{E}_{i}\right)^{\frac{2 \gamma}{1+\gamma}},
$$

where the error indicators $\mathcal{E}_{i}, i=0, \ldots, 5$, are defined by

$$
\begin{aligned}
& \mathcal{E}_{0}=\left\|u_{0}-U_{h}^{0}\right\|_{L^{1}(\Omega)} \\
& \mathcal{E}_{1}=\left(\sum_{n=1}^{m} \tau_{n}\left\|h_{n}^{1 / 2} \llbracket \nabla A\left(U_{h}^{n}\right) \rrbracket\right\|_{L^{2}(\Omega)}^{2}\right)^{1 / 2} \quad \text { jump residual, } \\
& \mathcal{E}_{2}=\left(\sum_{n=1}^{m} \tau_{n}\left\|h_{n} R^{n}\right\|_{L^{2}(\Omega)}^{2}\right)^{1 / 2} \quad \text { interior residual, } \\
& \mathcal{E}_{3}=\left(\sum_{n=1}^{m} \tau_{n}\left\|\nabla\left(A\left(U_{h}^{n}\right)-A\left(U_{h}^{n-1}\right)\right)\right\|_{L^{2}(\Omega)}^{2}\right)^{1 / 2} \quad \text { time residual, } \\
& \mathcal{E}_{4}=\sum_{n=1}^{m} \int_{t^{n-1}}^{t^{n}}\left\|\frac{U_{h}^{n}-\bar{U}_{h}^{n-1}}{\tau_{n}}-\left(\partial_{t} U_{h}+\operatorname{div} f\left(U_{h}\right)\right)\right\|_{L^{1}(\Omega)} d t \quad \text { characteristic } \\
& \mathcal{E}_{5}=\sum_{n=1}^{m} \int_{t^{n-1}}^{t^{n}}\left\|g-\bar{g}^{n}\right\|_{L^{1}(\Omega)} d t \quad \text { source. }
\end{aligned}
$$

Proof. In the proof we will make use of the Clément interpolation operator $\Pi^{n}$ : $H_{0}^{1}(\Omega) \rightarrow V_{0}^{n}$, which satisfies the following local approximation properties [9], for any $\varphi \in H_{0}^{1}(\Omega)$ :

$$
\begin{aligned}
& \left\|\varphi-\Pi^{n} \varphi\right\|_{L^{2}(K)}+h_{K}\left\|\nabla\left(\varphi-\Pi^{n} \varphi\right)\right\|_{L^{2}(K)} \leq C^{*} h_{K}\|\nabla \varphi\|_{L^{2}(N(K))}, \\
& \left\|\varphi-\Pi^{n} \varphi\right\|_{L^{2}(e)} \leq C^{*} h_{e}^{1 / 2}\|\nabla \varphi\|_{L^{2}(N(e))},
\end{aligned}
$$

where $N(A)$ is the union of all elements in $\mathcal{M}^{n}$ surrounding the sets $A=K \in \mathcal{M}^{n}$ or $A=e \in \mathcal{B}^{n}$. The constant $C^{*}$ depends only on the minimum angle of the mesh $\mathcal{M}^{n}$.

Denote $\zeta=H_{\varepsilon}\left(A\left(U_{h}\right)-A(u)\right) \theta$, where $\theta$ will be chosen later. Then by (4.2) and (3.4), we know that, for $t \in\left(t^{n-1}, t^{n}\right]$,

$$
\begin{aligned}
\langle\mathcal{R}, \zeta\rangle=\left\langle g-\bar{g}^{n}, \zeta\right\rangle+\left\langle R^{n}-\Delta A\left(U_{h}^{n}\right), \zeta-\Pi^{n} \zeta\right\rangle-\left\langle\nabla A\left(U_{h}^{n}\right), \nabla\left(\zeta-\Pi^{n} \zeta\right)\right\rangle \\
+\left\langle\frac{U_{h}^{n-1}-\bar{U}_{h}^{n-1}}{\tau_{n}}-\operatorname{div} f\left(U_{h}\right), \zeta\right\rangle-\left\langle\nabla\left(A\left(U_{h}\right)-A\left(U_{h}^{n}\right)\right), \nabla \zeta\right\rangle,
\end{aligned}
$$


where $\Delta A\left(U_{h}^{n}\right)$ is understood in element-wise sense. Thus, after integrating by parts, we get

$$
\begin{aligned}
& -\int_{0}^{T}\left\langle\mathcal{R}, H_{\varepsilon}\left(A(u)-A\left(U_{h}\right)\right) \theta\right\rangle d t \\
= & -\sum_{n=1}^{N} \int_{t^{n-1}}^{t^{n}}\left\langle g-\bar{g}^{n}, \zeta\right\rangle-\sum_{n=1}^{N} \int_{t^{n-1}}^{t^{n}}\left\langle R^{n}, \zeta-\Pi^{n} \zeta\right\rangle \\
& +\sum_{n=1}^{N} \int_{t^{n-1}}^{t^{n}} \sum_{e \in \mathcal{B}^{n}} \int_{e} \llbracket \nabla A\left(U_{h}^{n}\right) \rrbracket_{e}\left(\zeta-\Pi^{n} \zeta\right) \\
& -\sum_{n=1}^{N} \int_{t^{n-1}}^{t^{n}}\left\langle\frac{U_{h}^{n}-\bar{U}_{h}^{n-1}}{\tau_{n}}-\left(\partial_{t} U_{h}+\operatorname{div} f\left(U_{h}\right)\right), \zeta\right\rangle \\
& +\sum_{n=1}^{N} \int_{t^{n-1}}^{t^{n}}\left\langle\nabla\left(A\left(U_{h}\right)-A\left(U_{h}^{n}\right)\right), \nabla \zeta\right\rangle d t \\
=: & \mathrm{I}+\cdots+\mathrm{V} .
\end{aligned}
$$

Now we assume that $0 \leq \theta \leq 1$ and $\theta$ is supported in $\left(0, t^{m}\right)$. Since $H_{\varepsilon}(A(u)-$ $\left.A\left(U_{h}\right)\right) \leq 1$, we have

$$
\mathrm{I}+\mathrm{IV} \leq \mathcal{E}_{4}+\mathcal{E}_{5}
$$

By (5.12)-(5.13), we have

$$
\mathrm{II}+\mathrm{III} \leq C \sum_{n=1}^{m} \int_{t^{n-1}}^{t^{n}}\left(\left\|h_{n} R^{n}\right\|_{L^{2}(\Omega)}^{2}+\left\|h_{n}^{1 / 2} \llbracket \nabla A\left(U_{h}^{n}\right) \rrbracket\right\|_{L^{2}(\Omega)}^{2}\right)^{1 / 2}\|\nabla \zeta\|_{L^{2}(\Omega)} .
$$

Recall that $\nabla \zeta=H_{\varepsilon}^{\prime}\left(A\left(U_{h}\right)-A(u)\right) \nabla\left(A\left(U_{h}\right)-A(u)\right) \theta$. By Young's inequality,

$$
\mathrm{II}+\mathrm{III} \leq \frac{1}{4} \int_{Q} H_{\varepsilon}^{\prime}\left(A(u)-A\left(U_{h}\right)\right)\left|\nabla\left(A(u)-A\left(U_{h}\right)\right)\right|^{2} \theta+C \varepsilon^{-1}\left(\mathcal{E}_{1}+\mathcal{E}_{2}\right)^{2} .
$$

Similarly, we have

$$
\mathrm{V} \leq \frac{1}{4} \int_{Q} H_{\varepsilon}^{\prime}\left(A(u)-A\left(U_{h}\right)\right)\left|\nabla\left(A(u)-A\left(U_{h}\right)\right)\right|^{2} \theta+C \varepsilon^{-1}\left(\mathcal{E}_{3}\right)^{2}
$$

Substituting these estimates into (5.10) we arrive at, for any $\theta \in C_{c}^{\infty}\left(0, t^{m}\right)$ satisfying $0 \leq \theta \leq 1$,

$$
\begin{aligned}
-\int_{Q} U_{\varepsilon}\left(u, U_{h}\right) \theta_{t} \leq & \mathcal{K} \varepsilon^{\gamma} \int_{Q} \frac{1}{\nu\left(\varepsilon, U_{h}\right)}\left(\left|\partial_{t} U_{h}\right|+\left|\nabla_{x} U_{h}\right|\right) \theta \\
& +\left(\mathcal{E}_{4}+\mathcal{E}_{5}\right)+C \varepsilon^{-1}\left(\sum_{i=1}^{3} \mathcal{E}_{i}\right)^{2} .
\end{aligned}
$$

The following argument to choose $\theta$ is standard (see, e.g., 24]). For any $0<t_{1}<$ $t_{2}<t^{m}$, take $\alpha$ sufficiently small such that $t_{1}-\alpha>0, t_{2}+\alpha<t^{m}$, and define

$$
\theta(t)=\int_{t-t_{2}}^{t-t_{1}} \omega_{\alpha}(s) d s
$$


where $\omega_{\alpha}$ is the symmetric mollifier in $\mathbf{R}$. Then it is clear that $0 \leq \theta \leq 1$ and $\theta_{t}=\omega_{\alpha}\left(t-t_{1}\right)-\omega_{\alpha}\left(t-t_{2}\right)$. Thus

$$
\begin{aligned}
-\int_{Q} U_{\varepsilon}\left(u, U_{h}\right) \theta_{t} & =\int_{Q} U_{\varepsilon}\left(u, U_{h}\right) \omega_{\alpha}\left(t-t_{2}\right)-\int_{Q} U_{\varepsilon}\left(u, U_{h}\right) \omega_{\alpha}\left(t-t_{1}\right) \\
& \rightarrow \int_{\Omega} U_{\varepsilon}\left(u, U_{h}\right)\left(t_{2}\right) d x-\int_{\Omega} U_{\varepsilon}\left(u, U_{h}\right)\left(t_{1}\right) d x, \quad \text { as } \alpha \rightarrow 0 .
\end{aligned}
$$

From the definition of the entropy function $U_{\varepsilon}(z, k)=\int_{k}^{z} H_{\varepsilon}(A(r)-A(k)) d r$, it is easy to prove that

$$
|z-k|-\frac{\varepsilon}{\nu(\varepsilon, k)} \leq U_{\varepsilon}(z, k) \leq|z-k|
$$

where $\nu(\varepsilon, k)=\min \left\{A^{\prime}(s):|A(s)-A(k)| \leq \varepsilon\right\}$ is defined the same as in (5.1).

Thus

$$
\begin{aligned}
& -\lim _{\alpha \rightarrow 0} \int_{Q} U_{\varepsilon}\left(u, U_{h}\right) \theta_{t} \\
& \quad \geq\left\|\left(u-U_{h}\right)\left(t_{2}\right)\right\|_{L^{1}(\Omega)}-\left\|\left(u-U_{h}\right)\left(t_{1}\right)\right\|_{L^{1}(\Omega)}-\varepsilon \int_{\Omega} \frac{1}{\nu\left(\varepsilon, U_{h}\left(x, t_{2}\right)\right)} d x \\
& \quad \rightarrow\left\|u^{m}-U_{h}^{m}\right\|_{L^{1}(\Omega)}-\left\|u_{0}-U_{h}^{0}\right\|_{L^{1}(\Omega)}-\varepsilon\left\|\nu\left(\varepsilon, U_{h}^{m}\right)^{-1}\right\|_{L^{1}(\Omega)},
\end{aligned}
$$

as $t_{2} \rightarrow t^{m}$ and $t_{1} \rightarrow t^{0}$. Therefore we deduce from (5.14) that

$$
\begin{aligned}
\left\|u^{m}-U_{h}^{m}\right\|_{L^{1}(\Omega)} \leq & \varepsilon^{\gamma}\left(\mathcal{K} \int_{Q_{m}} \frac{1}{\nu\left(\varepsilon, U_{h}\right)}\left(\left|\partial_{t} U_{h}\right|+\nabla_{x} U_{h} \mid\right)+\int_{\Omega} \frac{1}{\nu\left(\varepsilon, U_{h}^{m}\right)}\right) \\
& +\mathcal{E}_{0}+\mathcal{E}_{4}+\mathcal{E}_{5}+C \varepsilon^{-1}\left(\sum_{i=1}^{3} \mathcal{E}_{i}\right)^{2} .
\end{aligned}
$$

Now let $\varepsilon_{0}=\left(\sum_{i=1}^{3} \mathcal{E}_{i}\right)^{\frac{2}{1+\gamma}}$ and take $\varepsilon=\varepsilon_{0} / \Lambda_{m}^{\frac{1}{1+\gamma}}$, where $\Lambda_{m}$ is defined in (5.11). Since $\varepsilon \leq \varepsilon_{0}$, we have $\nu\left(\varepsilon, U_{h}\right) \geq \nu\left(\varepsilon_{0}, U_{h}\right), \nu\left(\varepsilon, U_{h}^{m}\right) \geq \nu\left(\varepsilon_{0}, U_{h}^{m}\right)$, and consequently,

$$
\int_{Q_{m}} \frac{1}{\nu\left(\varepsilon, U_{h}\right)}\left(\left|\partial_{t} U_{h}\right|+\left|\nabla_{x} U_{h}\right|\right)+\int_{\Omega} \frac{1}{\nu\left(\varepsilon, U_{h}^{m}\right)} \leq \Lambda_{m}
$$

Thus

$$
\left\|u^{m}-U_{h}^{m}\right\|_{L^{1}(\Omega)} \leq \mathcal{E}_{0}+\mathcal{E}_{4}+\mathcal{E}_{5}+C \Lambda_{m}^{\frac{1}{1+\gamma}}\left(\sum_{i=1}^{3} \mathcal{E}_{i}\right)^{\frac{2 \gamma}{1+\gamma}} .
$$

This completes the proof.

To conclude this section, we give several remarks about the a posteriori error estimate derived in this section.

Remark 5.7. In practical computations, the error indicator $\mathcal{E}_{0}$ for the initial error can be easily reduced by refining the initial mesh, and the source error indicator $\mathcal{E}_{5}$ can be controlled by reducing time step sizes. The characteristic error indicator $\mathcal{E}_{4}$ can be reduced by reducing the time step size if the approximate characteristic $\tilde{X}(t)$ in (3.5) is solved by a convergent multistep Euler method or a high-order Runge-Kutta method. 
Remark 5.8. In the case of strong diffusion $A^{\prime}(s) \geq \beta>0$ for any $s \in \mathbf{R}$ and where $A^{\prime}$ is uniformly Lipschitz continuous, then the Hölder exponent $\gamma=1$ in (H2) and $\Lambda_{n}$ is bounded by $\beta^{-1}\left\|U_{h}\right\|_{B V\left(Q_{n}\right)}$, which is expected to be bounded in practical computations. The a posteriori error estimator in Theorem 5.6 then recovers the standard a posteriori error estimator derived in the literature for parabolic problems [30, 7. In particular, the space error indicators $\mathcal{E}_{1}^{n}, \mathcal{E}_{2}^{n}$, which control the adaptation of finite element meshes at each time step, are sharp in the sense that a local lower bound for the error can be established by extending the argument in 7, Theorem 2.2 ] for linear parabolic equations.

Remark 5.9. In the case of small constant viscosity $A^{\prime}=\epsilon$, then the Hölder exponent $\gamma=1$ in (H2), and $\Lambda_{m}=C \epsilon^{-1}$. The estimators derived in Theorem 5.6 are closely related to the estimators in 21], in which $L^{2}\left(L^{2}\right)$ a posteriori error estimates are derived based on the duality argument for the linear convection-dominated equation

$$
\frac{\partial u}{\partial t}+\operatorname{div}(\mathbf{v} u)-\epsilon \Delta u=g \quad \text { in } Q,
$$

where $\mathbf{v} \in C(\bar{Q})^{2}$ such that divv $=0$. For the linear problem (5.15), we remark that one can derive an $L^{\infty}\left(L^{1}\right)$ a posteriori error estimate of the same form as in Theorem [5.6 without using the Kružkov "doubling of variables" technique. We now describe briefly this simple argument. The weak formulation of (5.15) is

$$
\left\langle\partial_{t} u, \varphi\right\rangle-\langle\mathbf{v} u, \nabla \varphi\rangle+\epsilon\langle\nabla u, \nabla \varphi\rangle=\langle g, \varphi\rangle \quad \forall \varphi \in H_{0}^{1}(\Omega) .
$$

The discrete problem is the same as in (3.4), and we define the discrete residual $\mathcal{R} \in L^{2}\left(0, T ; H^{-1}(\Omega)\right)$ similar to (4.2), for any $\varphi \in H_{0}^{1}(\Omega)$ :

$$
\left\langle\partial_{t} U_{h}, \varphi\right\rangle-\left\langle\mathbf{v} U_{h}, \nabla \varphi\right\rangle+\epsilon\left\langle\nabla U_{h}, \nabla \varphi\right\rangle=\langle g, \varphi\rangle-\langle\mathcal{R}, \varphi\rangle .
$$

Subtracting (5.16) from (5.17) we get the following error equation, for any $\varphi \in$ $H_{0}^{1}(\Omega)$,

$$
\left\langle\partial_{t}\left(u-U_{h}\right), \varphi\right\rangle-\left\langle\mathbf{v}\left(u-U_{h}\right), \nabla \varphi\right\rangle+\epsilon\left\langle\nabla\left(u-U_{h}\right), \nabla \varphi\right\rangle=\langle\mathcal{R}, \varphi\rangle .
$$

The a posteriori error estimate can be readily derived by taking $\varphi=H_{\delta}\left(u-U_{h}\right)$, where $H_{\delta}(s)=s / \sqrt{s^{2}+\delta^{2}}$ is a regularization of $\operatorname{sgn}(s)$, using the following Galerkin orthogonality for $t \in\left(t^{n-1}, t^{n}\right]$ :

$$
\begin{aligned}
\langle\mathcal{R}, \varphi\rangle= & \left\langle g-\bar{g}^{n}, \varphi\right\rangle+\left\langle\frac{U_{h}^{n}-\bar{U}_{h}^{n-1}}{\tau_{n}}-\left(\partial_{t} U_{h}+\mathbf{v} \nabla U_{h}\right), \varphi\right\rangle \\
& +\left\langle R^{n}, \varphi-\Pi^{n} \varphi\right\rangle-\epsilon\left\langle\nabla U_{h}^{n}, \nabla\left(\varphi-\Pi^{n} \varphi\right)\right\rangle-\epsilon\left\langle\nabla\left(U_{h}-U_{h}^{n}\right), \nabla \varphi\right\rangle,
\end{aligned}
$$

and exploiting the standard argument in the a posteriori error analysis. We remark, however, that this simple argument cannot be extended to deal with the nonlinear problem considered in this paper.

Remark 5.10. The method of the a posteriori error analysis in this paper is different from those for nonlinear conservation laws in [11, 12, 25, or nonlinear degenerate parabolic equations in [28]. Recall that there are several parameters introduced in the analysis:

- The regularizing parameter $\varepsilon$ in $H_{\varepsilon}(z)$.

- The boundary layer sequence parameters $\delta, \eta$ and the mollifier parameters $l, m, n$. 
The analysis for Cauchy problems in [11, 12, 25] is based on letting $\varepsilon \rightarrow 0$ and taking finite mollifier parameters $l, m, n$. The analysis in [28] takes both finite $\varepsilon$ and finite mollifier parameters $l, m, n$. Note that there are no boundary layer sequence parameters $\delta, \eta$ for the analysis for Cauchy problems. The analysis in this paper is based on letting $\delta, \eta \rightarrow 0$ and $l, m, n \rightarrow \infty$ but taking a finite $\varepsilon$. We are not able to use the same technique as that in 11, 12, 25, 28, by choosing finite mollifier parameters $l, m, n$ to treat the problem with boundary conditions.

\section{NumericAl EXAMPLES}

The computation in this section makes use of the adaptive finite element toolbox ALBERT 32. In both examples we have $\gamma=1$ in (H2). Thus by Theorem 5.6, we know that

$$
\begin{aligned}
& \max _{1 \leq m \leq N}\left\|u^{m}-U_{h}^{m}\right\|_{L^{1}(\Omega)} \\
& \quad \leq \mathcal{E}_{0}+\sum_{n=1}^{N}\left(\mathcal{E}_{4}^{n}+\mathcal{E}_{5}^{n}\right)+C\left(\sum_{n=1}^{N} \tau_{n} \eta_{\text {time }}^{n}\right)^{1 / 2}+C\left(\sum_{n=1}^{N} \tau_{n} \eta_{\text {space }}^{n}\right)^{1 / 2},
\end{aligned}
$$

where

$$
\begin{aligned}
& \mathcal{E}_{4}^{n}=\int_{t^{n-1}}^{t^{n}}\left\|\frac{U_{h}^{n}-\bar{U}_{h}^{n-1}}{\tau_{n}}-\left(\partial_{t} U_{h}+\operatorname{div} f\left(U_{h}\right)\right)\right\|_{L^{1}(\Omega)} d t, \\
& \mathcal{E}_{5}^{n}=\int_{t^{n-1}}^{t^{n}}\left\|g-\bar{g}^{n}\right\|_{L^{1}(\Omega)} d t,
\end{aligned}
$$

and the time error indicator $\eta_{\text {time }}^{n}$ and space error indicator $\eta_{\text {space }}^{n}$ are given by

$$
\eta_{\text {time }}^{n}=\sum_{K \in \mathcal{M}^{n}} \Lambda_{K}^{n}\left\|\nabla\left(A\left(U_{h}^{n}\right)-A\left(U_{h}^{n-1}\right)\right)\right\|_{L^{2}(K)}^{2}, \quad \eta_{\text {space }}^{n}=\sum_{K \in \mathcal{M}^{n}} \eta_{K}^{n}
$$

with the local error indicator $\eta_{K}^{n}$ defined as

$$
\eta_{K}^{n}=\Lambda_{K}^{n}\left\|h_{K} R^{n}\right\|_{L^{2}(K)}^{2}+\sum_{e \subset \partial K} \Lambda_{e}^{n}\left\|h_{e}^{1 / 2} \llbracket \nabla A\left(U_{h}^{n}\right) \rrbracket\right\|_{L^{2}(e)}^{2} .
$$

Here $\Lambda_{K}^{n}$ and $\Lambda_{e}^{n}$ which are chosen according to the factor $\Lambda_{n}$ in (5.11) will be specified later. Let the time and space tolerances $\mathrm{TOL}_{\text {time }}$ and $\mathrm{TOL}_{\text {space }}$ be given. At each time step $n \geq 1$, the time step size $\tau_{n}$ is determined through the requirements

$$
\eta_{\text {time }}^{n} \leq \frac{\text { TOL }_{\text {time }}{ }^{2}}{4 T}, \quad \frac{1}{\tau_{n}}\left(\mathcal{E}_{4}^{n}+\mathcal{E}_{5}^{n}\right) \leq \frac{\text { TOL }_{\text {time }}}{2 T} .
$$

The set of elements marked for refinements $\mathcal{M}_{\text {refine }}^{n}$ and the set of elements marked for coarsening $\mathcal{M}_{\text {coarse }}^{n}$ are determined by the Guaranteed Error Reduction Strategy 14, [15]:

$$
\sum_{K \in \mathcal{M}_{\text {refine }}^{n}} \eta_{K}^{n} \geq \theta_{r}^{2} \sum_{K \in \mathcal{M}^{n}} \eta_{K}^{n}, \quad \sum_{K \in \mathcal{M}_{\text {coarse }}^{n}} \eta_{K}^{n} \leq \theta_{c}^{2} \sum_{K \in \mathcal{M}^{n}} \eta_{K}^{n}
$$

The iteration for the mesh adaptation at each time step $n$ is terminated whenever $\eta_{\text {space }}^{n} \leq \mathrm{TOL}_{\text {space }}{ }^{2} / T$ is satisfied. The elements in $\mathcal{M}_{\text {refine }}^{n}$ are chosen from those with largest error indicators, and the elements in $\mathcal{M}_{\text {coarse }}^{n}$ are taken from those with smallest error indicators (see [32, p. 36] for further implementation details). 
TABLE 6.1. The initial errors and the numbers of nodes of the initial meshes for different choices of $\epsilon$, TOL and TOL $\mathrm{L}_{\text {initial }}$ for Example 1.

\begin{tabular}{|c|c|c|c|c|}
\hline & TOL & TOL initial $_{\text {ind }}$ & $\mathcal{E}_{0}$ & number of nodes \\
\hline \multirow{4}{*}{$\epsilon=1 \mathrm{e}-5$} & 6.0 & $2.0 \mathrm{e}-4$ & $1.47 \mathrm{e}-4$ & 8415 \\
\cline { 2 - 5 } & 4.0 & $1.0 \mathrm{e}-4$ & $9.58 \mathrm{e}-5$ & 13407 \\
\cline { 2 - 5 } & 2.0 & $5.0 \mathrm{e}-5$ & $4.96 \mathrm{e}-5$ & 24751 \\
\cline { 2 - 5 }$\epsilon=1 \mathrm{e}-3$ & 1.0 & $2.5 \mathrm{e}-5$ & $1.77 \mathrm{e}-5$ & 68051 \\
\hline & 1.0 & $1.0 \mathrm{e}-3$ & $5.73 \mathrm{e}-4$ & 2247 \\
\cline { 2 - 5 } & 0.5 & $5.0 \mathrm{e}-4$ & $3.91 \mathrm{e}-4$ & 3287 \\
\cline { 2 - 5 } & 0.25 & $2.5 \mathrm{e}-4$ & $1.47 \mathrm{e}-4$ & 8415 \\
\cline { 2 - 5 } & 0.125 & $1.25 \mathrm{e}-4$ & $9.58 \mathrm{e}-5$ & 13407 \\
\hline
\end{tabular}

Example 1. We consider the so-called rotating cylinder problem from 21, Example 6.3] for the linear convection diffusion equation

$$
\frac{\partial u}{\partial t}+\operatorname{div}(\mathbf{v} u)-\epsilon \Delta u=g \quad \text { in } Q .
$$

Let $\Omega=(0,1)^{2}, T=0.5, g=0, \mathbf{v}=-2 \pi\left(2 x_{2}-1,1-2 x_{1}\right)^{T}$ and

$$
u_{0}= \begin{cases}1, & \text { for } s \leq 1 / 4, \\ 0, & \text { otherwise }\end{cases}
$$

where $s^{2}=\left(2 x_{1}-1 / 2\right)^{2}+\left(2 x_{2}-1\right)^{2}$. In the computations we take TOL $\mathrm{L}_{\text {time }}=\mathrm{TOL}_{\text {space }}$ and $\theta_{r}=0.5, \theta_{c}=0.1$. The initial mesh $\mathcal{M}_{0}$ at time $t=0$ is so chosen that $\mathcal{E}_{0}=$ $\left\|u_{0}-U_{h}^{0}\right\|_{L^{1}(\Omega)} \leq \mathrm{TOL}_{\text {initial }}$. In our computations, we take $\mathrm{TOL}_{\text {initial }} \ll \mathrm{TOL}_{\text {space }}$ so that the initial errors are negligible. Table 6.1 shows the initial errors and the numbers of nodes of the corresponding initial meshes for different choices of $\epsilon$, $\mathrm{TOL}_{\text {initial }}$, and the corresponding $\mathrm{TOL}=\mathrm{TOL}_{\text {space }}+\mathrm{TOL}_{\text {time }}$.

Since $A(s)=\epsilon s$, we have $\Lambda_{n} \leq C \epsilon^{-1}$ from the definition in (5.11) or by following the argument in Remark 5.9. Thus we take $\Lambda_{K}^{n}=\epsilon^{-1}$ and $\Lambda_{e}^{n}=\epsilon^{-1}$ in (6.1) and (6.2). Table 6.2 shows the total number of nodes $M=\sum_{n=1}^{N} M_{n}$, where $M_{n}$ is the number of nodes of $\mathcal{M}_{n}$, the total estimated error

$$
\eta=\sum_{n=1}^{N}\left(\mathcal{E}_{4}^{n}+\mathcal{E}_{5}^{n}\right)+\left(\sum_{n=1}^{N} \tau_{n} \eta_{\text {time }}^{n}\right)^{1 / 2}+\left(\sum_{n=1}^{N} \tau_{n} \eta_{\text {space }}^{n}\right)^{1 / 2}
$$

TABLE 6.2. The total number of nodes $M$, the total estimated error $\eta$, and the convergence rate $\alpha$ when $\epsilon=10^{-3}$ (left) and $\epsilon=10^{-5}$ (right) for Example 1 .

\begin{tabular}{|c|c|c|c|}
\hline TOL & $M$ & $\eta$ & $\alpha$ \\
\hline 1.0 & 113238 & 0.6392 & \\
\hline 0.5 & 728872 & 0.3267 & -0.3605 \\
\hline 0.25 & 4434790 & 0.1637 & -0.3827 \\
\hline 0.125 & 25173197 & 0.0814 & -0.4024 \\
\hline
\end{tabular}

\begin{tabular}{|c|c|c|c|}
\hline TOL & $M$ & $\eta$ & $\alpha$ \\
\hline 6.0 & 102111 & 3.7485 & \\
\hline 4.0 & 267457 & 2.6576 & -0.3572 \\
\hline 2.0 & 2046604 & 1.2973 & -0.3524 \\
\hline 1.0 & 14878263 & 0.6426 & -0.3541 \\
\hline
\end{tabular}


TABLE 6.3. The total number of nodes $M$, the total estimated error $\eta$, and the convergence rate $\alpha$ in the case of uniform refinements both in space and time when $\epsilon=10^{-3}$ (left) and $\epsilon=10^{-5}$ (right) for Example 1.

\begin{tabular}{|c|c|c|}
\hline$M$ & $\mathcal{E}$ & $\alpha$ \\
\hline 21130 & 1.1118 & \\
\hline 166420 & $7.8015 \mathrm{e}-1$ & -0.1716 \\
\hline 1321000 & $4.9420 \mathrm{e}-1$ & -0.2204 \\
\hline 10526800 & $3.0042 \mathrm{e}-1$ & -0.2398 \\
\hline
\end{tabular}

\begin{tabular}{|c|c|c|}
\hline$M$ & $\mathcal{E}$ & $\alpha$ \\
\hline 21130 & 2.8175 & \\
\hline 166420 & 2.0943 & -0.1437 \\
\hline 1321000 & 1.5442 & -0.1471 \\
\hline 10526800 & 1.1249 & -0.1527 \\
\hline
\end{tabular}

and the convergence rate $\alpha$ for $\epsilon=10^{-3}$ and $\epsilon=10^{-5}$, respectively. For two different $\mathrm{TOL}_{i}$, let $\eta_{i}$ and $M_{i}$ be the corresponding total estimated error and total number of nodes, and the convergence rate $\alpha$ is computed by $\alpha=\log \left(\eta_{1} / \eta_{2}\right) / \log \left(M_{1} / M_{2}\right)$.

We observe from Tables 6.2 that the total estimated error $\eta$ is roughly proportional to $M^{-1 / 3}$, i.e., $\eta \approx C M^{-1 / 3}$ for some constant $C>0$. This indicates that

$$
\max _{1 \leq n \leq N}\left\|u-U^{n}\right\|_{L^{1}(\Omega)} \leq C M^{-1 / 3}
$$

We observe from Table 6.3 that because of the singular nature of the solutions, the numerical scheme (3.4)-(3.5) with uniform refinements both in space and time does not produce the convergence rate $\eta \approx C M^{-1 / 3}$ in terms of the error reduction.

Figure 6.1 shows the meshes and the surface plots of the solutions at time $t=$ 0.251278 and $t=0.500878$ when $\epsilon=10^{-5}$. We observe that the meshes "follow" the positions of the cylinder. For this problem, the "leakage" of the numerical solutions is observed in 21 in the following sense: the mesh is coarser in the regions of the cylinder closest to and farthest from the center of rotation. We do not observe, however, this phenomenon in our computation as indicated in Figure 6.1. This may be explained by the difference of the error indicators used in two papers.

Example 2. Let the domain $\Omega=(0,1)^{2}$ and $T=0.5$. We consider the Richards equation (1.3)

$$
\frac{\partial S}{\partial t}+\frac{\partial K(S)}{\partial x_{1}}-\operatorname{div}(K(S) \nabla p)=0 \quad \text { in } Q
$$

with the initial condition $S(x, 0)=\min \left(1, \max \left(0.5,1-64 x_{1}\right)\right)$ and the boundary condition $S(x, t)=S(x, 0)$. The constitutive relations are taken as in (1.4) with shape constants $\alpha=1$ and $n=1.3$.

Notice that for the nonlinear diffusion, the factor $\Lambda_{m}$ in (5.11) depends on the discrete solution $U_{h}$ in the whole time interval which makes the adaptive algorithm impractical if we take $\Lambda_{K}^{n}$ and $\Lambda_{e}^{n}$ in (6.1) (6.2) to be equal to $\Lambda_{n}$. In our computations, motivated by the algorithm for constant viscosity in Example 1, we take

$$
\Lambda_{K}^{n}=\max _{x \in N(K)} A^{\prime}\left(U_{h}^{n}(x)\right)^{-1}, \quad \Lambda_{e}^{n}=\max _{x \in N(e)} A^{\prime}\left(U_{h}^{n}(x)\right)^{-1},
$$

where $N(A)$ is the union of all elements in $\mathcal{M}^{n}$ surrounding the sets $A=K \in \mathcal{M}^{n}$ or $A=e \in \mathcal{B}^{n}$. 

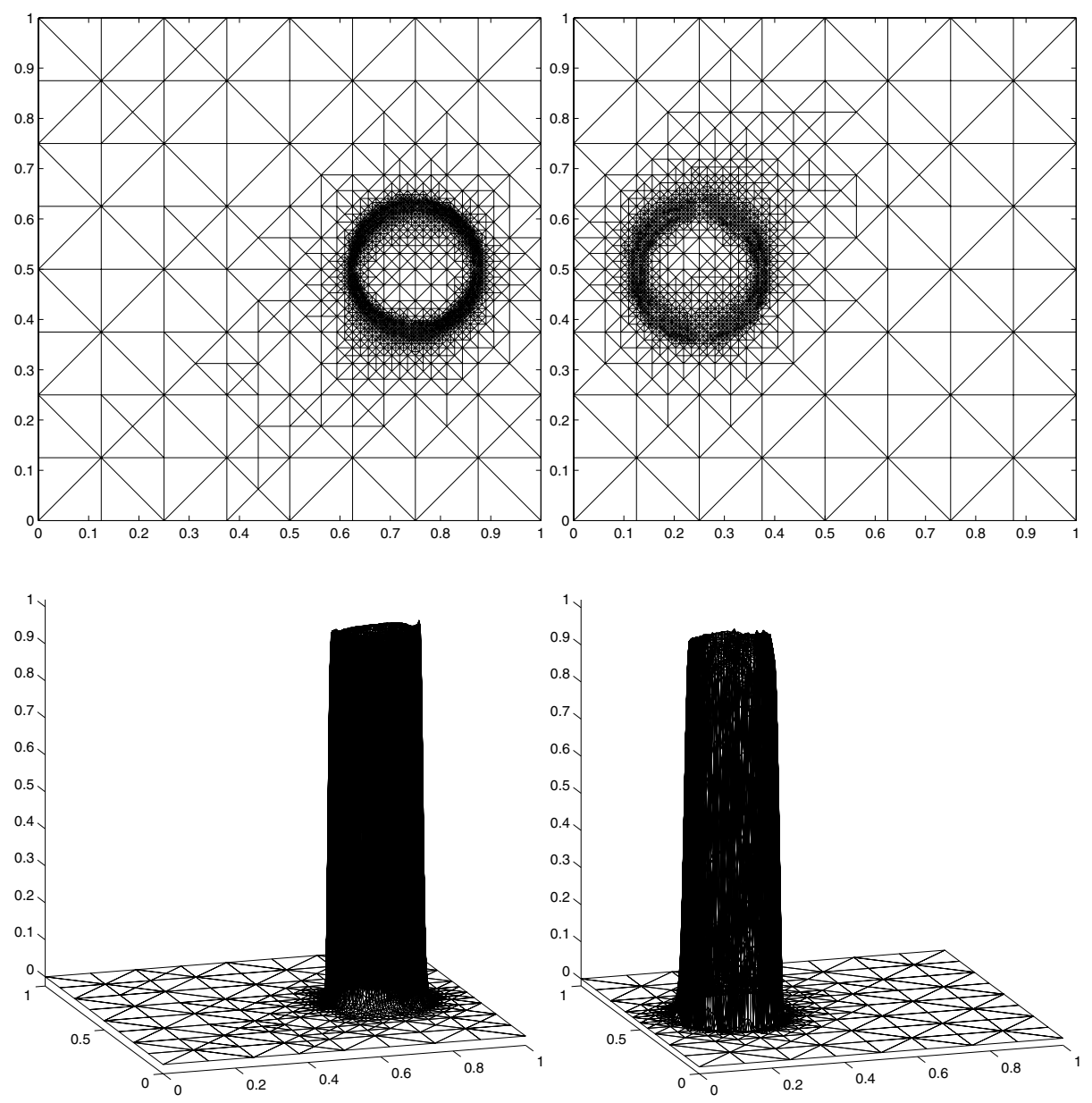

Figure 6.1. The meshes (top) and the surface plots (bottom) of the solutions at time $t=0.251278$ (left) and $t=0.500878$ (right) when $\epsilon=10^{-5}$ for Example 1. The number of nodes are 3133 $(t=0.251278)$ and $2143(t=0.500878)$, respectively.

Table 6.4 shows the total number of nodes $M=\sum_{n=1}^{N} M_{n}$, where $M_{n}$ is the number of nodes of $\mathcal{M}_{n}$, the total estimated error

$$
\eta=\sum_{n=1}^{N}\left(\mathcal{E}_{4}^{n}+\mathcal{E}_{5}^{n}\right)+\Lambda_{N}^{1 / 2}\left(\sum_{n=1}^{N} \tau_{n} \mathcal{E}^{n}\right)^{1 / 2}
$$

and the convergence rate $\alpha$, where

$$
\mathcal{E}^{n}=\left\|h_{n}^{1 / 2} \llbracket \nabla A\left(U_{h}^{n}\right) \rrbracket\right\|_{L^{2}(\Omega)}^{2}+\left\|h_{n} R^{n}\right\|_{L^{2}(\Omega)}^{2}+\left\|\nabla\left(A\left(U_{h}^{n}\right)-A\left(U_{h}^{n-1}\right)\right)\right\|_{L^{2}(\Omega)}^{2} .
$$

We observe from Table 6.4 that the total estimated error $\eta$ is roughly proportional to $M^{-1 / 3}$, i.e., $\eta \approx C M^{-1 / 3}$ for some constant $C>0$. This rather satisfatory property shows the competitiveness of our adaptive algorithm for the problem considered. 
TABLE 6.4. The total number of nodes $M$, the total estimated error $\eta$, and the convergence rate $\alpha$ for Example 2 .

\begin{tabular}{|c|c|c|c|c|}
\hline TOL $_{\text {space }}$ & TOL $_{\text {time }}$ & $M$ & $\eta$ & $\alpha$ \\
\hline 10 & 0.5 & 3322791 & 36.1525 & \\
\hline 5 & 0.25 & 10997806 & 22.0889 & -0.4116 \\
\hline 2.5 & 0.125 & 37323710 & 12.6322 & -0.4573 \\
\hline 1.5 & 0.075 & 96129806 & 8.3865 & -0.4330 \\
\hline 1.25 & 0.0625 & 162752230 & 7.2182 & -0.3800 \\
\hline
\end{tabular}

Figure 6.2 shows the meshes and the surface plots of the solutions at time $t=0.1$ and $t=0.3$ when $\mathrm{TOL}_{\text {space }}=1.25$. We observe that the meshes "follow" the positions where the solutions change most. Also the meshes are rather fine near the upper and lower part of the boundary as a consequence of the boundary layer effect. We will report more implementation details and numerical experiments for the nonlinear convection-diffusion problems in a forthcoming paper.
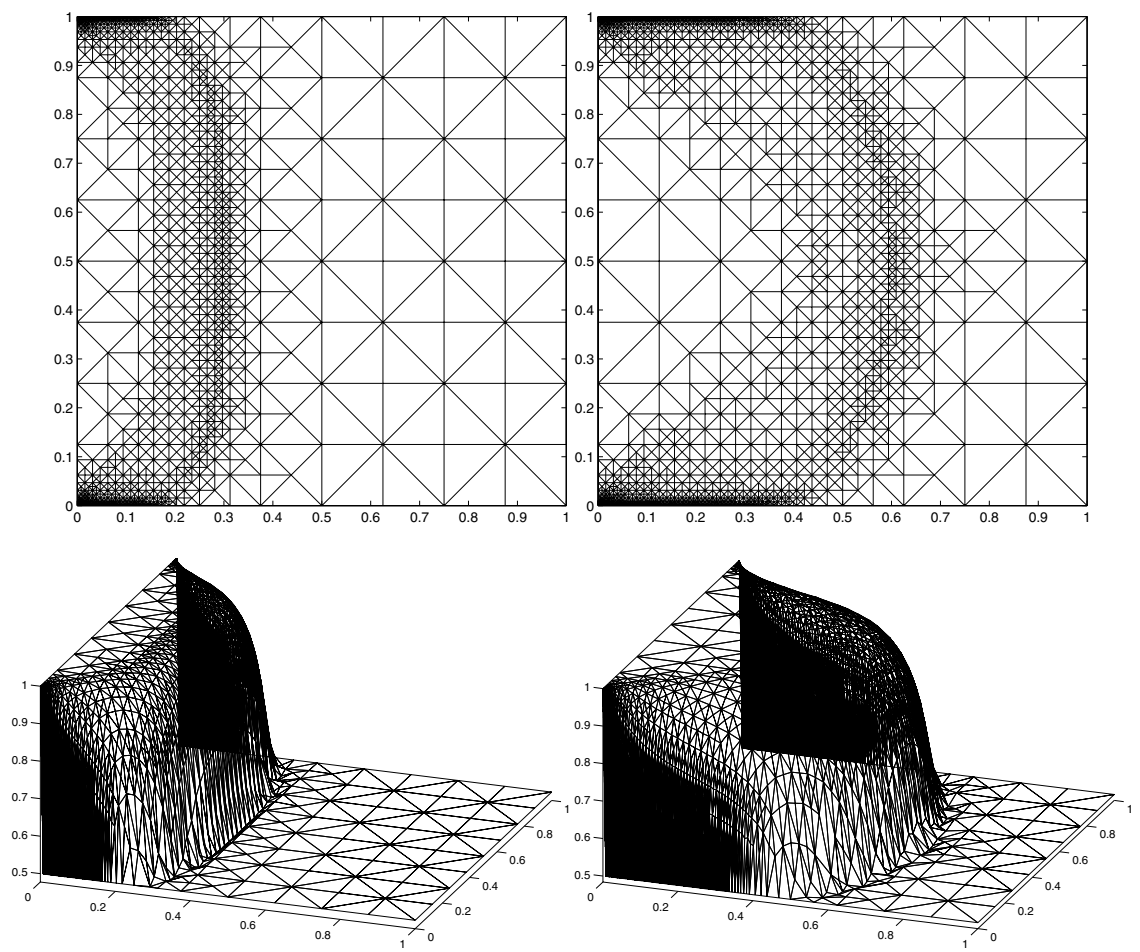

Figure 6.2. The meshes (top) and the surface plots (bottom) of the solutions at time $t=0.1$ (left) and $t=0.3$ (right) for Example 2. The number of nodes are $2595(t=0.1)$ and $3733(t=0.3)$, respectively. 


\section{ACKNOWLEDGMENTS}

The authors would like to thank the referees for the careful reading of the manuscript and for their constructive comments.

\section{REFERENCES}

[1] T. Arbogast and M.F. Wheeler, A characteristic-mixed finite element method for advectiondominated transport problems, SIAM J. Numer. Anal. 32 (1995), 404-424. MR1324295 (95m:65151)

[2] H.W. Alt and S. Luckhaus, Quasilinear elliptic-parabolic differential equations, Math. Z. 183 (1983), 311-341. MR0706391 (85c:35059)

[3] I. Babuška and C. Rheinboldt, Error estimates for adaptive finite element computations, SIAM J. Numer. Anal. 15 (1978), 736-754. MR0483395 (58:3400)

[4] J. Carrillo, Entropy solutions for nonlinear degenerate problems, Arch. Rational Mech. Anal. 147 (1999), 269-361. MR.1709116 (2000m:35132)

[5] Z. Chen and S. Dai, Adaptive Galerkin methods with error control for a dynamical GinzburgLandau model in superconductivity, SIAM J. Numer. Anal. 38 (2001), 1961-1985. MR.1856238 (2002g:65114)

[6] Z. Chen and S. Dai. On the efficiency of adaptive finite element methods for elliptic problems with discontinuous coefficients, SIAM J. Sci. Comput. 24 (2002), 443-462. MR1951050 (2003k:65160)

[7] Z. Chen and F. Jia, An adaptive finite element method with reliable and efficient error control for linear elliptic problems, Math. Comp. 73 (2004), 1163-1197. MR2047083

[8] Z. Chen, R.H. Nochetto and A. Schmidt, A characteristic Galerkin method with adaptive error control for the continuous casting problem, Comput. Methods Appl. Mech. Engrg. 189 (2000), 249-276. MR1779683 (2001f:80003)

[9] Ph. Clément, Approximation by finite element functions using local regularization, RAIRO Anal. Numer. 9 (1975), 77-84. MR0400739(53:4569)

[10] B. Cockburn, A simple introduction to error estimation for nonlinear hyperbolic conservation laws, Department of Mathematics, University of Minnesota, 2003. http://www.math.umn.edu/ cockburn/LectureNotes.html

[11] B. Cockburn, F. Coquel and P.G. Lefloch, An error estimate for finite volume methods for multidimensional conservation laws, Math. Comp. 63 (1994), 77-103. MR1240657 (95d:65078)

[12] B. Cockburn and P.-A. Gremaud, Error estimates for finite element methods for scalar conservation laws, SIAM J. Numer. Anal. 33 (1996), 522-554. MR1388487(97e:65096)

[13] C.N. Dawson, T.F. Russell and M.F. Wheeler, Some improved error estimates for the modified method of characteristics, SIAM J. Numer. Anal. 26 (1989), 1487-1512. MR1025101 (91a:65229)

[14] W. Dörfler, A convergent adaptive algorithm for Possion's equations, SIAM J. Numer. Anal. 33 (1996), 1106-1124. MR 1393904 (97e:65139)

[15] W. Dörfler, A time and space adaptive algorithm for the linear time-dependent Schrödinger equation, Numer. Math. 73 (1996), 419-448. MR1393174 (97g:65183)

[16] J. Douglas, Jr. and T.F. Russell, Numerical methods for convection-dominated diffusion problem based on combining the method of characteristics with finite element or finite difference procedures, SIAM J. Numer. Anal. 19 (1982), pp. 871-885. MR0672564 (84b:65093)

[17] K. Eriksson and C. Johnson, Adaptive finite element methods for parabolic problems I: A linear model problem, SIAM J. Numer. Anal. 28 (1991), 43-77. MR.1083324 (91m:65274)

[18] R. Eymard, T. Gallouët and R. Herbin, Error estimate for approximate solutions of a nonlinear convection-diffusion problem, Adv. Differ. Equ. 7 (2002), 419-440. MR 1869118 (2002h:35156)

[19] R. Eymard, T. Gallouët, R. Herbin and A. Michel, Convergence of a finite volume scheme for nonlinear degenerate parabolic equations, Numer. Math. 92 (2002), 41-82. MR 1917365 (2003e:65159)

[20] C. Johnson and A. Szepessy, Adaptive finite element methods for conservation laws based on a posteriori error estimates, Comm. Pure Appl. Math. XLVIII (1995), 199-234. MR1322810 (97b:76084) 
[21] P. Houston and E. Süli, Adaptive Lagrange-Galerkin methods for unsteady convectiondiffusion problems, Math. Comp. 70 (2000), 77-106. MR1681108(2001f:65114)

[22] K. Huang, R. Zhang and M.T. van Genuchten, An Eulerian-Lagrangian approach with an adaptively corrected method of characteristic to simulate variably saturated water flow, Water Resource Research 30 (1994), 499-507.

[23] J. Kačur, Solution of degenerate convection-diffusion problems by the method of characteristics, SIAM J. Numer. Anal. 39 (2001), 858-879. MR1860448(2002h:65144)

[24] K.H. Karlsen and N.H. Risebro, On the uniqueness and stability of entropy solutions of nonlinear degenerate parabolic equations with rough coefficients, Discrete Contin. Dynam. Systems - Series A 9 (2003), 1081-1104. MR1974417 (2004h:35102)

[25] D. Kröner and M. Ohlberger, A posteriori error estimates for upwind finite volume schemes for nonlinear conservation laws in multi-dimensions, Math. Comp. 69 (2000), 25-39. MR:1659843 (2000i:65135)

[26] N.N. Kružkov, First order quasi-linear equations in several independent variables, Math. USSR Sbornik 10 (1970), 217-243. MR0267257(42:2159)

[27] C. Mascia, A. Porretta and A. Terracina, Nonhomogeneous Dirichlet problems for degenerate parabolic-hyperbolic equations, Arch. Rational Mech. Anal. 163 (2002), 87-124. MR1911095 (2003e:35217)

[28] M. Ohlberger, A posteriori error estimates for vertex centered finite volume approximations of convection-diffusion-reaction equations, Math. Model. Numer. Anal. 35 (2001), 355-387. MR1825703 (2002a:65142)

[29] F. Otto, $L^{1}$-contraction and uniqueness for quasilinear elliptic-parabolic equations, J. Diff. Equations 131 (1996), 20-38. MR1415045 (97i:35125)

[30] M. Picasso, Adaptive finite elements for a linear parabolic problem, Comput. Methods Appl. Mech. Engrg. 167 (1998), 223-237. MR:1673951(2000b:65188)

[31] O. Pironneau, On the transport-diffusion algorithm and its application to the Navier-Stokes equations, Numer. Math. 38 (1982), 309-332. MR0654100 (83d:65258)

[32] A. Schmidt and K.G. Siebert, ALBERT: An adaptive hierarchical finite element toolbox, IAM, University of Freiburg, 2000. http://www.mathematik.unifreiburg.de/IAM/Research/projectsdz/albert.

LSEC, Institute of Computational Mathematics, Academy of Mathematics and System Sciences, Chinese Academy of Sciences, Beijing 100080, People's Republic of China

E-mail address: zmchen@lsec.cc.ac.cn

Institute of Computational Mathematics, Academy of Mathematics and System Sciences, Chinese Academy of Sciences, Beijing 100080, People's Republic of China

E-mail address: ghji@lsec.cc.ac.cn 